\title{
Free vibration analysis of piezoelectric cylindrical nanoshell: nonlocal and surface elasticity effects
}

\author{
SAYYID H. HASHEMI KACHAPI \\ Department of Mechanical Engineering \\ Babol Noshirvani University of Technology \\ Shariati Street, Babol, Mazandaran47148-71167 \\ IRAN \\ sha.hashemi.kachapi@gmail.com
}

\begin{abstract}
Vibration analysis of piezoelectric cylindrical nanoshell subjected to visco-Pasternak medium with arbitrary boundary conditions is investigated. In these analysis simultaneous effects of the nonlocal, surface elasticity and the different material scale parameter are considered. To this end, Eringen nonlocal theory and Gurtin-Murdoch surface/interface theory considering Donnell's shell theory are used. The governing equations and boundary conditions are derived using Hamilton's principle and the assumed mode method combined with Euler-Lagrange method is used for discretizing the equations of motion. The viscoelastic nanoshell medium is modeled as Visco-Pasternak foundation. A variety of new vibration results including frequencies and mode shapes for piezoelectric cylindrical nano-shell with non-classical restraints as well as different material parameters are presented. The convergence, accuracy and reliability of the current formulation are validated by comparisons with existing experimental and numerical results. Also, the effects of nonlocality, surface energy, nanoshell radius, circumferential wavenumber, nanoshell damping coefficient, and foundation damping are accurately studied on frequencies and mode shapes of piezoelectric cylindrical nanoshell.
\end{abstract}

Key-Words: - Piezoelectric nanoshell, Gurtin-Murdoch surface/interface theory, Eringen nonlocal theory, visco-Pasternak medium, natural frequency.

Received: October 22, 2019. Revised: March 21, 2020. Accepted: April 20, 2020. Published: May 7, 2020.

\section{Introduction}

Recently, with the development of material science, nano-sized piezoelectric elements such as nanobeams, nano-membranes and nano-shells have been fabricated, and are attracting worldwide attention in nano-electro-mechanical (NEM) devices [1-3]. Since the classical continuum theory is scale-free, it fails to predict the size-dependent response of nano-structures. Consequently, to consider the small scale effect, some non-classical continuum theories such as couple stress theory [4], nonlocal elasticity theory [5-6], strain gradient theory [7], and surface elasticity theory [8-9] have been introduced to develop the size-dependent continuum models. The electro-elastic surface/interface theory expanded from GurtinMurdoch elasticity theory has been used to analyze the surface and the size dependent vibration of piezoelectric nano-structures [10-14]. In the past two decades, investigating the nano-sized and surface effects on the mechanical behavior of nanostructures have become one of the attractive research areas in nanomechanics, as evidenced by the large number of publications on this issue [1523]. The nonlinear buckling and postbuckling behaviors of shear deformable nano-shell under radial compressive load were studied by using the surface elasticity theory [16]. With considering the surface elasticity theory, vibration analysis of fluidconveying nanotubes was presented by Wang [18]. Surface effects on the dispersion characteristics of elastic waves propagating in an infinite piezoelectric nanoplate are investigated by Zhang et al. based on the surface piezoelectric constitutive theory [19]. Also the nonlinear free vibration and nonlinear postbuckling behaviors of nano-plates were studied by Wang et al. [20]. Theoretical formulation based on Sanders' thin shell theory for the natural frequencies of vibration of functionally graded cylindrical shells is established by Rahimi et al. [21]. Avramov presented nonlinear vibration and bifurcation behavior of single-walled carbon nanotubes using the Sanders-Koiter shell theory, 
nonlocal elasticity, Galerkin and the harmonic balanced methods [22]. Nonlinear harmonic vibration of a piezoelectric-layered nanotube conveying fluid flow is investigated by Saadatnia et al. using the nonlocal theory and energy approach [23]. Also the frequency-amplitude relationship and the frequency response of the system are studied. In an excellent monograph by Leissa [24], researches on the vibration analysis of thin shells before 1970s were reviewed. There are also more recent survey such as Liew et al. which review articles about vibration analysis of shallow shells [25]. Loy et al. presented the free vibration analysis of cylindrical shells using an improved version of the differential quadrature method [26]. According to surface/interface and small scale effects, recently, Hashemi Kachapi et al. investigated linear and nonlinear vibration and stability analysis of multiwalled piezoelectric nanostructures [27-37].

In the present study, the free vibration analysis of a piezoelectric cylindrical nanoshell subjected to Visco-Pasternak medium with arbitrary boundary conditions is investigated usinf Eringen nonlocal theory and the Gurtin-Murdoch surface/interface theory. A variety of new vibration results including the effects of nonlocality, surface energy, nanoshell radius, circumferential wavenumber, nanoshell damping coefficient, and foundation damping with non-classical restraints are accurately studied on frequencies and mode shapes of piezoelectric cylindrical nanoshell.

\section{Problem formulation and governing equations}

A cylindrical nano shell embedded with a piezoelectric layer and visco-Pasternak medium is shown in Figure 1. The nano shell has length of $L$, mid-surface radius $R$, thickness of $2 h_{N}$, and piezoelectric layer thickness of $h_{p}$. With the origin of coordinate system located on the middle surface of nano-shell, the coordinates of a typical point in the axial, circumferential and radius directions are described by $x, \theta$, and $z$, respectively. Also, $K_{w}, K_{p}$ and $C_{w}$ are stiffness coefficient of Winkler foundation, shear layer of Pasternak foundation and the damping factor of the visco-Pasternak medium for the transverse motion, respectively. $E_{N}, v_{N}$ and
$\rho_{N}$ represent Young modulus, Poisson ratio and the mass density of cylindrical nano-shell. It is assumed that the material properties nano-shell vary through the thickness according to the power law function. They are written as:

$$
\begin{aligned}
& E_{N}=\left(E_{o}-E_{I}\right)\left(\frac{2 z+h_{N}}{2 h_{N}}\right)^{q}+E_{I} \\
& v_{N}=\left(v_{o}-v_{I}\right)\left(\frac{2 z+h_{N}}{2 h_{N}}\right)^{q}+v_{I} \\
& \rho_{N}=\left(\rho_{o}-\rho_{I}\right)\left(\frac{2 z+h_{N}}{2 h_{N}}\right)^{q}+\rho_{I}
\end{aligned}
$$

where $q$ is the power-law exponent. The subscripts $O$ and $I$ represent the properties of the nano-shell at the outer and inner surfaces, respectively.

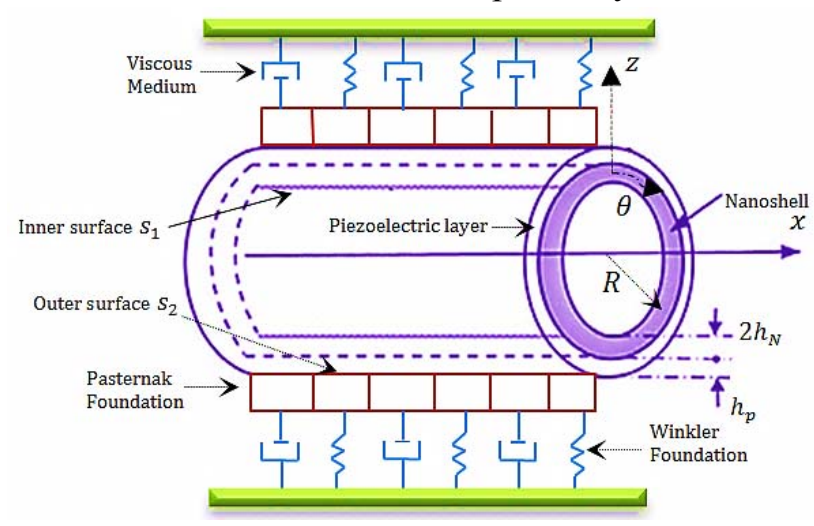

Fig. 1. A piezoelectric cylindrical nano shell with inner and outer surfaces

Young modulus, Poisson ratio, piezoelectric and dielectric constants and also the mass density of piezoelectric layer are respectively expressed as $E_{p}$, $v_{p}, e_{31 p}, e_{32 p}, \eta_{33 p}$ and $\rho_{p}$. The surface at the outer piezoelectric layer is denoted by $s_{2}$, and the inner surface is denoted by $s_{1}$. The material properties of surface $s_{2}$ are Lamé's constants $\lambda^{s_{2}}$, $\mu^{s_{2}}$ residual stress $\tau_{0}^{s_{2}}$ and piezoelectric constants $e_{31 p}^{s_{2}}$ and $e_{32 p}^{s_{2}}$. Those of the inner surface are Lamé's constants $\lambda^{s_{1}}, \mu^{s_{1}}$ and residual stress $\tau_{0}^{s_{1}}$.

Due to the character of nano-shell, the state of generalized plane stress of shells is assumed, and the normal stress in the radial direction is zero. In the cylindrical nano-shell, the constitutive relation can be expressed as $[37,38]$;

$$
\begin{aligned}
\left\{\begin{array}{l}
\sigma_{x x N} \\
\sigma_{\theta \theta N} \\
\tau_{x \theta N}
\end{array}\right\}= & {\left[\begin{array}{ccc}
C_{11 N} & C_{12 N} & 0 \\
C_{21 N} & C_{22 N} & 0 \\
0 & 0 & C_{66 N}
\end{array}\right]\left\{\begin{array}{l}
\varepsilon_{x x} \\
\varepsilon_{\theta \theta} \\
\gamma_{x \theta}
\end{array}\right\}, } \\
\text { or } & \left\{\sigma_{N}\right\}=\left[C_{N}\right]\{\varepsilon\},
\end{aligned}
$$


In the outside piezoelectric shell, the constitutive relation can be expressed as $[37,38]$

$$
\begin{aligned}
& \left\{\begin{array}{l}
\sigma_{x x p} \\
\sigma_{\theta \theta p} \\
\tau_{x \theta p}
\end{array}\right\}=\left[\begin{array}{ccc}
C_{11 p} & C_{12 p} & 0 \\
C_{21 p} & C_{22 p} & 0 \\
0 & 0 & C_{66 p}
\end{array}\right]\left\{\begin{array}{l}
\varepsilon_{x x} \\
\varepsilon_{\theta \theta} \\
\gamma_{x \theta}
\end{array}\right\} \\
& -\left[\begin{array}{ccc}
0 & 0 & e_{31 p} \\
0 & 0 & e_{32 p} \\
0 & 0 & 0
\end{array}\right]\left\{\begin{array}{l}
\bar{E}_{x p} \\
\bar{E}_{\theta p} \\
\bar{E}_{z p}
\end{array}\right\}, \\
& \text { or }\left\{\sigma_{p}\right\}=\left[C_{p}\right]\{\varepsilon\}-\left[e_{p}\right]\left\{\bar{E}_{p}\right\},
\end{aligned}
$$

in which the subscripts $N$ and $P$ represent the cylindrical nano-shell and piezoelectric layers, respectively. $\left\{\bar{E}_{p}\right\}$ is the vector of electric field for piezoelectric layers. $\left[C_{N}\right]$ and $\left[C_{p}\right]$ are the matrices of elastic constants with following elements.

$$
\begin{aligned}
& C_{11 N}=\frac{E_{N}}{1-v_{N}^{2}}=C_{22 N}, \\
& C_{12 N}=\frac{v_{N} E_{N}}{1-v_{N}^{2}}=C_{21 N}, \\
& C_{66 N}=\frac{E_{N}}{2\left(1+v_{N}\right)} \\
& C_{11 p}=\frac{E_{p}}{1-v_{p}^{2}}=C_{22 p}, \\
& C_{12 p}=\frac{v_{p} E_{p}}{1-v_{p}^{2}}=C_{21 p}, \\
& C_{66 p}=\frac{E_{p}}{2\left(1+v_{p}\right)}
\end{aligned}
$$

Since the piezoelectric layers are very thin, $\bar{E}_{x p}$ and $\bar{E}_{\theta p}$ are assumed to be zero $\left(\bar{E}_{x p}=\bar{E}_{\theta p}=0\right)$, and only the radial component of electric field $\bar{E}_{z p}$ is considered. Consequently, $\left\{\bar{E}_{p}\right\}$ can be written as [39]

$$
\left\{\begin{array}{lll}
\bar{E}_{x p} & \bar{E}_{\theta p} & \bar{E}_{z p}
\end{array}\right\}^{T}=\left\{\begin{array}{lll}
0 & 0 & V_{p} / h_{p}
\end{array}\right\}^{T},
$$

where $V_{p}$ is the voltage applied to piezoelectric layers. In addition, the voltages at the piezoelectric surface $S_{2}\left(z=h_{N}+h_{p}\right)$ and $S_{1}\left(z=h_{N}\right)$ are $+V_{p}$ and $-V_{p}$, respectively. Based on these assumptions, the radial component of electric displacement $D_{z p}$ can be presented as

$D_{z p}=e_{31 p} \varepsilon_{x x}+e_{32 p} \varepsilon_{\theta \theta}+\eta_{33 p} \bar{E}_{z p}$

\subsection{Non- classical Shell theory (Gurtin- Murdoch surface/interface theory)}

Within the framework of classical shell theory, the displacement fields of the nano-shell can be written as [38]

$$
\begin{aligned}
& u_{x}(x, \theta, z)=u(x, \theta)-z \frac{\partial w(x, \theta)}{\partial x}, \\
& u_{\theta}(x, \theta, z)=v(x, \theta)-\frac{z}{R} \frac{\partial w(x, \theta)}{\partial \theta}, \\
& u_{z}(x, \theta, z)=w(x, \theta),
\end{aligned}
$$

where $u, v$ and $w$ stand for the middle surface displacements in the $x, \theta$ and $z$ directions, respectively. The linear deflection and curvatures are defined by Donnell's theory as [37, 38]

$$
\begin{aligned}
& \left\{\begin{array}{l}
\varepsilon_{x x} \\
\varepsilon_{\theta \theta} \\
\gamma_{x \theta}
\end{array}\right\}=\left\{\begin{array}{l}
\varepsilon_{x x}^{0} \\
\varepsilon_{\theta \theta}^{0} \\
\gamma_{x \theta}^{0}
\end{array}\right\}+z\left\{\begin{array}{l}
\kappa_{x x} \\
\kappa_{\theta \theta} \\
\kappa_{x \theta}
\end{array}\right\} \\
& =\left\{\begin{array}{c}
\frac{\partial u}{\partial x} \\
\frac{1}{R} \frac{\partial v}{\partial \theta}+\frac{1}{R} w \\
\frac{1}{R} \frac{\partial u}{\partial \theta}+\frac{\partial v}{\partial x}
\end{array}\right\}-z\left\{\begin{array}{c}
\frac{\partial^{2} w}{\partial x^{2}} \\
\frac{1}{R^{2}} \frac{\partial^{2} w}{\partial \theta^{2}} \\
\frac{2}{R} \frac{\partial^{2} w}{\partial x \partial \theta}
\end{array}\right\}
\end{aligned}
$$

in which $\varepsilon_{x x}^{0}, \varepsilon_{\theta \theta}^{0}$ and $\gamma_{x \theta}^{0}$ are the middle surface strains, and $\kappa_{x x}, \kappa_{\theta \theta}$ and $\kappa_{x \theta}$ are the curvature components of the nano-shell.

Due to the nano-sized property, the ratio of surface to the volume becomes large, and the surface energy around the shell imposes significant effect on the vibration of nano-structure and the surface effect needs to be considered. Based on the GurtinMurdoch surface/interface theory, the constitute relations for surfaces can be written as [8-10].

$\sigma_{\alpha \beta}^{s_{2}}=\tau_{0}^{s_{2}} \delta_{\alpha \beta}+\left(\tau_{0}^{s_{2}}+\lambda^{s_{2}}\right) \varepsilon_{q q} \delta_{\alpha \beta}$

$+2\left(\mu^{s_{2}}-\tau_{0}^{s_{2}}\right) \varepsilon_{\alpha \beta}+\tau_{0}^{s_{2}} u_{\alpha, \beta}^{s_{2}}-e_{p}^{s_{2}} E_{z p}$,

$\sigma_{\alpha z}^{s_{2}}=\tau_{0}^{s_{2}} u_{z, \alpha}^{s_{2}}$

$\sigma_{\alpha z}^{s_{1}}=\tau_{0}^{s_{1}} u_{z, \alpha}^{s_{1}}(\alpha, \beta=x, \theta)$

$\sigma_{\alpha \beta}^{s_{1}}=\tau_{0}^{s_{1}} \delta_{\alpha \beta}+\left(\tau_{0}^{s_{1}}+\lambda^{s_{1}}\right) \varepsilon_{q q} \delta_{\alpha \beta}$

$+2\left(\mu^{s_{1}}-\tau_{0}^{s_{1}}\right) \varepsilon_{\alpha \beta}+\tau_{0}^{s_{1}} u_{\alpha, \beta}^{s_{1}}$,

in which $\delta_{\alpha \beta}$ is the Kronecker delta. Furthermore, the components of stress at the surfaces can be expressed as

$$
\begin{aligned}
& \sigma_{x x}^{s_{2}}=\left(\lambda^{s_{2}}+2 \mu^{s_{2}}\right) \varepsilon_{x x}+\left(\tau_{0}^{s_{2}}+\lambda^{s_{2}}\right) \varepsilon_{\theta \theta} \\
& +\tau_{0}^{s_{2}}-e_{31 p}^{s_{2}} E_{z p} \\
& \sigma_{\theta \theta}^{s_{2}}=\left(\tau_{0}^{s_{2}}+\lambda^{s_{2}}\right) \varepsilon_{x x}+\left(\lambda^{s_{2}}+2 \mu^{s_{2}}\right) \varepsilon_{\theta \theta} \\
& -\tau_{0}^{s_{2}} \frac{w}{R}+\tau_{0}^{s_{2}}-e_{32 p}^{s_{2}} E_{z p} \\
& \sigma_{x \theta}^{s_{2}}=\mu^{s_{2}} \gamma_{x \theta}-\tau_{0}^{s_{2}}\left(\frac{\partial v}{\partial x}-\frac{z}{R} \frac{\partial^{2} w}{\partial x \partial \theta}\right), \\
& \sigma_{\theta x}^{s_{2}}=\mu^{s_{2}} \gamma_{x \theta}-\tau_{0}^{s_{2}}\left(\frac{1}{R} \frac{\partial u}{\partial \theta}-\frac{z}{R} \frac{\partial^{2} w}{\partial x \partial \theta}\right)
\end{aligned}
$$


$\sigma_{x z}^{s_{2}}=\tau_{0}^{s_{2}} \frac{\partial w}{\partial x}, \quad \sigma_{\theta z}^{s_{2}}=\frac{\tau_{0}^{s_{2}}}{R} \frac{\partial w}{\partial \theta}$

$\sigma_{x z}^{s_{1}}=\tau_{0}^{s_{1}} \frac{\partial w}{\partial x}, \quad \sigma_{\theta z}^{s_{1}}=\frac{\tau_{0}^{s_{1}}}{R} \frac{\partial w}{\partial \theta}$,

$\sigma_{x x}^{s_{1}}=\left(\lambda^{s_{1}}+2 \mu^{s_{1}}\right) \varepsilon_{x x}+\left(\tau_{0}^{s_{1}}+\lambda^{s_{1}}\right) \varepsilon_{\theta \theta}$

$+\tau_{0}^{s_{1}}$

$\sigma_{\theta \theta}^{s_{1}}=\left(\tau_{0}^{s_{1}}+\lambda^{s_{1}}\right) \varepsilon_{x x}+\left(\lambda^{s_{1}}+2 \mu^{s_{1}}\right) \varepsilon_{\theta \theta}$

$-\tau_{0}^{s_{1}} \frac{w}{R}+\tau_{0}^{s_{1}}$

$\sigma_{x \theta}^{s_{1}}=\mu^{s_{1}} \gamma_{x \theta}-\tau_{0}^{s_{1}}\left(\frac{\partial v}{\partial x}-\frac{z}{R} \frac{\partial^{2} w}{\partial x \partial \theta}\right)$,

$\sigma_{\theta x}^{s_{1}}=\mu^{s_{1}} \gamma_{x \theta}-\tau_{0}^{s_{1}}\left(\frac{1}{R} \frac{\partial u}{\partial \theta}-\frac{z}{R} \frac{\partial^{2} w}{\partial x \partial \theta}\right)$,

Based on the classical continuum models, $\sigma_{z z}$ is neglected due to its small value as compared to other normal stress components. But, in the nonclassical continuum model, this assumption does not satisfy the surface conditions. Thus, it is supposed that $\sigma_{z z}$ varies linearly through the thickness and satisfies the balance conditions on the surfaces [40], i. e.

$\sigma_{z z}=$

$\frac{1}{2}\left(\begin{array}{c}\left(\frac{\partial \sigma_{x z}^{s_{2}}}{\partial x}+\frac{1}{R} \frac{\partial \sigma_{\theta z}^{s_{2}}}{\partial \theta}-\rho^{s_{2}} \frac{\partial^{2} w}{\partial t^{2}}\right) \\ -\left(\frac{\partial \sigma_{x z}^{s_{1}}}{\partial x}+\frac{1}{R} \frac{\partial \sigma_{\theta z}^{s_{1}}}{\partial \theta}-\rho^{s_{1}} \frac{\partial^{2} w}{\partial t^{2}}\right)\end{array}\right)$

$+\frac{1}{2 h_{N}+h_{p}}\left(\begin{array}{c}\left(\begin{array}{c}\frac{\partial \sigma_{x z}^{s_{2}}}{\partial x}+\frac{1}{R} \frac{\partial \sigma_{\theta z}^{s_{2}}}{\partial \theta} \\ -\rho^{s_{2}} \frac{\partial^{2} w}{\partial t^{2}}\end{array}\right) \\ +\left(\begin{array}{c}\frac{\partial \sigma_{x z}^{s_{1}}}{\partial x}+\frac{1}{R} \frac{\partial \sigma_{\theta z}^{s_{1}}}{\partial \theta} \\ -\rho^{s_{1}} \frac{\partial^{2} w}{\partial t^{2}}\end{array}\right)\end{array}\right) z$

By means of Eqs. (15) and (16), $\sigma_{z z}$ can be rewritten as

$\sigma_{z z}=\left(\begin{array}{c}\frac{\left(\tau_{0}^{s_{2}}-\tau_{0}^{s_{1}}\right)}{2} \\ +\frac{z\left(\tau_{0}^{s_{2}}+\tau_{0}^{s_{1}}\right)}{2 h_{N}+h_{p}}\end{array}\right) \times$

$\left(\frac{\partial^{2} w}{\partial x^{2}}+\frac{1}{R^{2}} \frac{\partial^{2} w}{\partial \theta^{2}}\right)$

$+\left(\frac{\left(\rho^{s_{1}}-\rho^{s_{2}}\right)}{2}-\frac{z\left(\rho^{s_{1}}+\rho^{s_{2}}\right)}{2 h_{N}+h_{p}}\right) \frac{\partial^{2} w}{\partial t^{2}}$,

According to Eq. (17), from Eqs. (4) and (5) the normal stresses $\sigma_{x x}$ and $\sigma_{\theta \theta}$ can be rewritten as

$$
\begin{aligned}
& \sigma_{x x N}=C_{11 N} \varepsilon_{x x}+C_{12 N} \varepsilon_{\theta \theta}+\frac{v_{N} \sigma_{z z}}{1-v_{N}}, \\
& \sigma_{\theta \theta N}=C_{21 N} \varepsilon_{x x}+C_{22 N} \varepsilon_{\theta \theta}+\frac{v_{N} \sigma_{z z}}{1-v_{N}}, \\
& \sigma_{x \theta N}=C_{66 N} \gamma_{x \theta}, \\
& \sigma_{x x p}=C_{11 p} \varepsilon_{x x}+C_{12 p} \varepsilon_{\theta \theta}-e_{31 p} \bar{E}_{x p} \\
& +\frac{v_{p} \sigma_{z z}}{1-v_{p}}, \\
& \sigma_{\theta \theta p}=C_{21 p} \varepsilon_{x x}+C_{22 p} \varepsilon_{\theta \theta}-e_{32 p} \bar{E}_{\theta p} \\
& +\frac{v_{N} \sigma_{z z}}{1-v_{p}}, \\
& \sigma_{x \theta p}=C_{66 p} \gamma_{x \theta},
\end{aligned}
$$

\subsubsection{Governing equations}

In this section, the governing equations of motion of the piezoelectric cylindrical nanoshell are obtained by applying the assumed mode method. The total strain energy considering the surface stress effect is expressed as:

$$
\begin{aligned}
& \pi=\frac{1}{2} \int_{0}^{L} \int_{0}^{2 \pi} \int_{-h_{N}}^{h_{N}}\left(\sigma_{i j N} \varepsilon_{i j}\right) R d z d \theta d x \\
& +\frac{1}{2} \int_{0}^{L} \int_{0}^{2 \pi} \int_{h_{N}}^{h_{N}+h_{p}}\left(\begin{array}{c}
\sigma_{i j p} \varepsilon_{i j} \\
-\bar{E}_{z p} D_{z p}
\end{array}\right) R d z d \theta d x \\
& +\frac{1}{2} \int_{0}^{L} \int_{0}^{2 \pi} \begin{array}{l}
\left(\sigma_{i j}^{S_{2}} \varepsilon_{i j}-\bar{E}_{z p} D_{i}^{S_{2}}\right) \times d \theta d x \\
\left(R+h_{N}+h_{p}\right)
\end{array} \\
& +\frac{1}{2} \int_{0}^{L} \int_{0}^{2 \pi}\left(\sigma_{i j}^{s_{1}} \varepsilon_{i j}\right)\left(R-h_{N}\right) d \theta d x=\frac{1}{2} \\
& \int_{0}^{L} \int_{0}^{2 \pi}\left\{\begin{array}{c}
N_{x x} \varepsilon_{x x}^{0}+N_{\theta \theta} \varepsilon_{\theta \theta}^{0} \\
+N_{x \theta} \gamma_{x \theta}^{0}+M_{x x} \kappa_{x x} \\
+M_{\theta \theta} \kappa_{\theta \theta}+M_{x \theta} \kappa_{x \theta} \\
+\eta_{33} \bar{E}_{z p}^{2} h_{p}
\end{array}\right\} R d \theta d x .
\end{aligned}
$$

In addition, the kinetic energy of the nanoshell can be formulated as:

$T=$

$\frac{1}{2} \int_{0}^{L} \int_{0}^{2 \pi}\left\{I\left(\begin{array}{c}\left(\frac{\partial u}{\partial t}\right)^{2}+\left(\frac{\partial v}{\partial t}\right)^{2} \\ +\left(\frac{\partial w}{\partial t}\right)^{2}\end{array}\right)\right\} R d \theta d x$

where

$$
\begin{aligned}
& I=\int_{-h_{N}}^{h_{N}} \rho_{N} d z+\int_{h_{N}}^{h_{N}+h_{p}} \rho_{p} d z \\
& +\left.\rho^{S_{2}}\right|_{z=-h_{N}}+\left.\rho^{S_{1}}\right|_{z=h_{N}+h_{p}}
\end{aligned}
$$


where $\rho_{N}, \rho_{p}$ and $\rho^{s}$ are the mass density of nanoshell, piezoelectric layer and surfaces, respectively.

The work done by the viscous damping and the applied elastic medium load, modeled using spring Winkler and shear Pasternak constants, respectively, can be expressed as

$W_{c}=-\frac{1}{2} \int_{0}^{L} \int_{0}^{2 \pi}\left\{C_{w}\left(\frac{\partial w}{\partial t}\right)^{2}\right\} R d \theta d x$

$W_{w p}=-\int_{0}^{L} \int_{0}^{2 \pi}\left\{\left(\begin{array}{c}K_{w} w \\ -K_{p} \nabla^{2} w\end{array}\right) w\right\} R d \theta d x$

in which $C_{w}, \nabla^{2}, K_{w}$ and $K_{p}$ are the damping coefficient of the viscoelastic medium, the Laplace operator, Winkler modulus, and the shear modulus of surrounding elastic medium, respectively [41]. Also, $\nabla^{2}=\frac{\partial^{2}}{\partial x^{2}}+\frac{1}{R^{2}} \frac{\partial^{2}}{\partial \theta^{2}}$.

In Eq. (20), the stresses and moment resultants are defined as:

$$
\begin{aligned}
& \left(N_{x x}, N_{\theta \theta}, N_{x \theta}\right)=\int_{-h_{N}}^{h_{N}} \sigma_{i j N} d z \\
& +\int_{h_{N}}^{h_{N}+h_{p}} \sigma_{i j p} d z+\sigma_{s_{1}}+\sigma_{S_{2}} \\
& =\left(N_{x N}, N_{\theta N}, N_{x \theta N}\right)+\left(N_{x p}, N_{\theta p}, N_{x \theta p}\right) \\
& +\left(\sigma_{x x}, \sigma_{\theta \theta}, \frac{1}{2}\left(\sigma_{x \theta}+\sigma_{\theta x}\right)\right)_{s_{1}} \\
& +\left(\sigma_{x x}, \sigma_{\theta \theta}, \frac{1}{2}\left(\sigma_{x \theta}+\sigma_{\theta x}\right)\right)_{s_{2}} \\
& \left(M_{x x}, M_{\theta \theta}, M_{x \theta}\right)=\int_{-h_{N}}^{h_{N}} \sigma_{i j N} z d z \\
& +\int_{h_{N}}^{h_{N}+h_{p}} \sigma_{i j p} z d z+\sigma_{S_{2}}\left(h_{N}+h_{p}\right)-\sigma_{s_{1}} h_{N} \\
& =\left(M_{x N}, M_{\theta N}, M_{x \theta N}\right)+\left(M_{x p}, M_{\theta p}, M_{x \theta p}\right) \\
& +\left(\sigma_{x x}, \sigma_{\theta \theta}, \frac{1}{2}\left(\sigma_{x \theta}+\sigma_{\theta x}\right)\right)_{s_{2}}\left(h_{N}+h_{p}\right) \\
& -\left(\sigma_{x x}, \sigma_{\theta \theta}, \frac{1}{2}\left(\sigma_{x \theta}+\sigma_{\theta x}\right)\right)_{s_{1}} h_{N} \\
& N_{x x}=A_{11} \varepsilon_{x x}^{0}+A_{12} \varepsilon_{\theta \theta}^{0}+B_{11} \kappa_{x x}+B_{12} \kappa_{\theta \theta} \\
& +\left(\tau_{0}^{s_{1}}+\tau_{0}^{s_{2}}-N_{x p}\right) \\
& +F_{11}^{*}\left(\frac{\partial^{2} w}{\partial x^{2}}+\frac{1}{R^{2}} \frac{\partial^{2} w}{\partial \theta^{2}}\right)+J_{11}^{*} \frac{\partial^{2} w}{\partial t^{2}}, \\
& N_{\theta \theta}=A_{21} \varepsilon_{x x}^{0}+A_{22} \varepsilon_{\theta \theta}^{0}+B_{21} \kappa_{x x}+B_{22} \kappa_{\theta \theta} \\
& -\frac{\left(\tau_{0}^{s_{1}}+\tau_{0}^{s_{2}}\right)}{R} w+\left(\tau_{0}^{s_{1}}+\tau_{0}^{s_{2}}-N_{\theta p}\right)
\end{aligned}
$$

in which

$$
\begin{aligned}
& A_{i j}=A_{i j N}+A_{i j p}+A_{i j}^{*}, \\
& B_{i j}=B_{i j N}+B_{i j p}+B_{i j}^{*}, \\
& D_{i j}=D_{i j N}+D_{i j p}+D_{i j}^{*}, \\
& F_{11}^{*}=F_{11 N}^{*}+F_{11 p}^{*}, E_{11}^{*}=E_{11 N}^{*}+E_{11 p}^{*},
\end{aligned}
$$

and

$$
\begin{aligned}
& \left(A_{i j N}, B_{i j N}, D_{i j N}\right)=\int_{-h_{N}}^{h_{N}} C_{i j N}\left(1, z, z^{2}\right) d z, \\
& \left(A_{i j p}, B_{i j p}, D_{i j p}\right)
\end{aligned}
$$$$
=\int_{h_{N}}^{h_{N}+h_{p}} C_{i j p}\left(1, z, z^{2}\right) d z \text {, }
$$$$
\left(N_{x p}, N_{\theta p}\right)=\int_{h_{N}}^{h_{N}+h_{p}}\left(e_{31 p}, e_{32 p}\right) \bar{E}_{z p} d z
$$$$
+\left(e_{31 p}^{s}, e_{32 p}^{s}\right) \bar{E}_{z p} \text {, }
$$$$
\left(M_{x p}, M_{\theta p}\right)=\int_{h_{N}}^{h_{N}+h_{p}}\left(e_{31 p}, e_{32 p}\right) \bar{E}_{z p} z d z
$$$$
+\left(e_{31 p}^{s}, e_{32 p}^{s}\right) \bar{E}_{z p}\left(h_{N}+h_{p}\right) \text {, }
$$$$
A_{11}^{*}=A_{22}^{*}=\left(\lambda^{s_{1}}+2 \mu^{s_{1}}\right)
$$$$
+\left(\lambda^{s_{2}}+2 \mu^{s_{2}}\right) \text {, }
$$$$
A_{12}^{*}=A_{21}^{*}=\left(\tau_{0}^{s_{1}}+\lambda^{s_{1}}\right)+\left(\tau_{0}^{S_{2}}+\lambda^{s_{2}}\right) \text {, }
$$$$
A_{66}^{*}=\left(\mu^{s_{1}}-\frac{\tau_{0}^{s_{1}}}{2}\right)+\left(\mu^{s_{2}}-\frac{\tau_{0}^{s_{2}}}{2}\right) \text {, }
$$$$
B_{11}^{*}=B_{22}^{*}=\left(\lambda^{s_{2}}+2 \mu^{s_{2}}\right)\left(h_{N}+h_{p}\right)
$$

$-\left(\lambda^{s_{1}}+2 \mu^{s_{1}}\right)\left(h_{N}\right)$,

$B_{12}^{*}=B_{21}^{*}=\left(\tau_{0}^{s_{2}}+\lambda^{s_{2}}\right)\left(h_{N}+h_{p}\right)$

$-\left(\tau_{0}^{s_{1}}+\lambda^{s_{1}}\right)\left(h_{N}\right)$,

$B_{66}^{*}=\left(\mu^{s_{2}}-\frac{\tau_{0}^{s_{2}}}{2}\right)\left(h_{N}+h_{p}\right)$ 
$-\left(\mu^{s_{1}}-\frac{\tau_{0}^{s_{1}}}{2}\right)\left(h_{N}\right)$,

$D_{11}^{*}=D_{22}^{*}=\left(\lambda^{s_{2}}+2 \mu^{s_{2}}\right)\left(h_{N}+h_{p}\right)^{2}$

$+\left(\lambda^{s_{1}}+2 \mu^{s_{1}}\right)\left(h_{N}\right)^{2}$,

$D_{12}^{*}=D_{21}^{*}=\left(\tau_{0}^{s_{2}}+\lambda^{s_{2}}\right)\left(h_{N}+h_{p}\right)^{2}$

$+\left(\tau_{0}^{s_{1}}+\lambda^{s_{1}}\right)\left(h_{N}\right)^{2}$,

$D_{66}^{*}=\left(\mu^{s_{2}}-\frac{\tau_{0}^{s_{2}}}{2}\right)\left(h_{N}+h_{p}\right)^{2}$

$+\left(\mu^{s_{1}}-\frac{\tau_{0}^{s_{1}}}{2}\right)\left(h_{N}\right)^{2}$,

$F_{11 N}^{*}=$

$\int_{-h_{N}}^{h_{N}} \frac{v_{N}}{\left(1-v_{N}\right)}\left(\begin{array}{c}\frac{\left(\tau_{0}^{s_{2}}-\tau_{0}^{s_{1}}\right)}{2} \\ +\frac{\left(\tau_{0}^{s_{2}}+\tau_{0}^{s_{1}}\right) z}{2 h_{N}+h_{p}}\end{array}\right) d z$,

$F_{11 p}^{*}=$

$\int_{h_{N}}^{h_{N}+h_{p}} \frac{v_{p}}{\left(1-v_{p}\right)}\left(\begin{array}{c}\frac{\left(\tau_{0}^{s_{2}}-\tau_{0}^{s_{1}}\right)}{2} \\ +\frac{\left(\tau_{0}^{s_{2}}+\tau_{0}^{s_{1}}\right) z}{2 h_{N}+h_{p}}\end{array}\right) d z$,

$J_{11 N}^{*}=$

$\int_{-h_{N}}^{h_{N}} \frac{v_{N}}{\left(1-v_{N}\right)}\left(\begin{array}{c}\frac{\left(\rho^{s_{1}}-\rho^{s_{2}}\right)}{2} \\ -\frac{\left(\rho^{s_{1}}+\rho^{s_{2}}\right) z}{2 h_{N}+h_{p}}\end{array}\right) d z$,

$J_{11 p}^{*}=$

$\int_{h_{N}}^{h_{N}+h_{p}} \frac{v_{p}}{\left(1-v_{p}\right)}\left(\begin{array}{c}\frac{\left(\rho^{s_{1}}-\rho^{s_{2}}\right)}{2} \\ -\frac{\left(\rho^{s_{1}}+\rho^{s_{2}}\right) z}{2 h_{N}+h_{p}}\end{array}\right) d z$,

$E_{11 N}^{*}=$

$\int_{-h_{N}}^{h_{N}} \frac{v_{N}}{\left(1-v_{N}\right)}\left(\begin{array}{c}\frac{\left(\tau_{0}^{s_{2}}-\tau_{0}^{s_{1}}\right) z}{2} \\ +\frac{\left(\tau_{0}^{s_{2}}+\tau_{0}^{s_{1}}\right) z^{2}}{2 h_{N}+h_{p}}\end{array}\right) d z$,

$E_{11 p}^{*}=$

$\int_{h_{N}}^{h_{N}+h_{p}} \frac{v_{p}}{\left(1-v_{p}\right)}\left(\begin{array}{c}\frac{\left(\tau_{0}^{s_{2}}-\tau_{0}^{s_{1}}\right) z}{2} \\ +\frac{\left(\tau_{0}^{s_{2}}+\tau_{0}^{s_{1}}\right) z^{2}}{2 h_{N}+h_{p}}\end{array}\right) d z$,

$G_{11 N}^{*}=$
$\int_{-h_{N}}^{h_{N}} \frac{v_{N}}{\left(1-v_{N}\right)}\left(\begin{array}{c}\frac{\left(\rho^{s_{1}}-\rho^{s_{2}}\right) z}{2} \\ -\frac{\left(\rho^{s_{1}}+\rho^{s_{2}}\right) z^{2}}{2 h_{N}+h_{p}}\end{array}\right) d z$,

$G_{11 p}^{*}=$

$\int_{h_{N}}^{h_{N}+h_{p}} \frac{v_{p}}{\left(1-v_{p}\right)}\left(\begin{array}{c}\frac{\left(\rho^{s_{1}}-\rho^{s_{2}}\right) z}{2} \\ -\frac{\left(\rho^{s_{1}}+\rho^{s_{2}}\right) z^{2}}{2 h_{N}+h_{p}}\end{array}\right) d z$,

Note that, because of geometric symmetry, the expressions $B_{i j N}$ and $F_{11 N}^{*}$ are zero, i.e. $\left(B_{i j N}=\right.$ $\left.F_{11 N}^{*}=0\right)$ and also, for free vibration of piezoelectric nanoshell $V_{p}$ is zero, as a result $\left(N_{x p}=N_{\theta p}=M_{x p}=M_{\theta p}=0\right)$.

In following, the equations of motion and corresponding boundary conditions of the piezoelectric nano shell can be derived from Hamilton's principle. By taking the variations of displacements $u, v$ and $w$, integrating by parts, and equating the coefficients of $\delta u$, $\delta v$ and $\delta w$ to zero, the governing equations of motion are derived as:

$\delta u: \frac{\partial N_{x x}}{\partial x}+\frac{1}{R} \frac{\partial N_{x \theta}}{\partial \theta}=I \frac{\partial^{2} u}{\partial t^{2}}$,

$\delta v: \frac{\partial N_{x \theta}}{\partial x}+\frac{1}{R} \frac{\partial N_{\theta \theta}}{\partial \theta}=I \frac{\partial^{2} v}{\partial t^{2}}$

$\delta w: \frac{\partial^{2} M_{x x}}{\partial x^{2}}+\frac{2}{R} \frac{\partial^{2} M_{x \theta}}{\partial x \partial \theta}+\frac{1}{R^{2}} \frac{\partial^{2} M_{\theta \theta}}{\partial \theta^{2}}$

$-\frac{N_{\theta \theta}}{R}+K_{p}\left(\frac{\partial^{2} w}{\partial x^{2}}+\frac{1}{R^{2}} \frac{\partial^{2} w}{\partial \theta^{2}}\right)$

$=I \frac{\partial^{2} w}{\partial t^{2}}+C_{w} \frac{\partial w}{\partial t}+K_{w} w$,

and boundary conditions are obtained as follows:

$$
\begin{aligned}
& \delta u=0 \quad \text { or } \quad N_{x x} n_{x}+\frac{1}{R} N_{x \theta} n_{\theta}=0, \\
& \delta v=0 \quad \text { or } \quad N_{x \theta} n_{x}+\frac{1}{R} N_{\theta \theta} n_{\theta}=0, \\
& \delta w=0 \quad \text { or } \\
& \left(\frac{\partial M_{x x}}{\partial x}+\frac{1}{R} \frac{\partial M_{x \theta}}{\partial \theta}\right) n_{x} \\
& +\left(\frac{1}{R} \frac{\partial M_{x \theta}}{\partial x}+\frac{1}{R^{2}} \frac{\partial M_{\theta \theta}}{\partial \theta}\right) n_{\theta}=0, \\
& \frac{\partial w}{\partial x}=0 \quad \text { or } \quad M_{x x} n_{x}+\frac{1}{R} M_{x \theta} n_{\theta}=0,
\end{aligned}
$$

$$
\frac{\partial w}{\partial \theta}=0 \text { or } \frac{1}{R} M_{x \theta} n_{x}+\frac{1}{R^{2}} M_{\theta \theta} n_{\theta}=0,
$$


In this paper, the assumed mode method is used to obtain the equations of motion using EulerLagrange method. After substituting Eqs. (25) into strain and kinetic energies Eqs. (20) and (21) and using following dimensionless parameters

$$
\begin{aligned}
& \bar{u}=\frac{u}{h_{N}}, \bar{v}=\frac{v}{h_{N}}, \bar{w}=\frac{w}{h_{N}}, \xi=\frac{x}{L}, \\
& \bar{b}=\frac{b}{L}, \bar{A}_{i j N}=\frac{A_{i j N}}{A_{11 N}}, \bar{B}_{i j N}=\frac{B_{i j N}}{A_{11 N} h_{N}}, \\
& \bar{D}_{i j N}=\frac{D_{i j N}}{A_{11 N} h_{N}^{2}}, \bar{A}_{i j p}=\frac{A_{i j p}}{A_{11 N}}, \\
& \bar{A}_{i j}^{*}=\frac{A_{i j}^{*}}{A_{11 N}}, \bar{B}_{i j p}=\frac{B_{i j p}}{A_{11 N} h_{N}}, \\
& \bar{B}_{i j}^{*}=\frac{B_{i j}^{*}}{A_{11 N} h_{N}}, \bar{D}_{i j p}=\frac{D_{i j p}}{A_{11 N} h_{N}^{2}}, \\
& \bar{D}_{i j}^{*}=\frac{D_{i j}^{*}}{A_{11 N} h_{N}^{2}}, \bar{F}_{11 N}^{*}=\frac{F_{11 N}^{*}}{A_{11 N} h_{N}}, \\
& \bar{F}_{11 p}^{*}=\frac{F_{11 p}^{*}}{A_{11 N} h_{N}}, \bar{E}_{11 N}^{*}=\frac{E_{11 N}^{*}}{A_{11 N} h_{N}^{2}}, \\
& \bar{E}_{11 p}^{*}=\frac{E_{11 p}^{*}}{A_{11 N} h_{N}^{2}}, \bar{J}_{11 N}^{*}=\frac{J_{11 N}^{*}}{\rho_{N} h_{N}^{2}}, \\
& \bar{J}_{11 p}^{*}=\frac{J_{11 p}^{*}}{\rho_{N} h_{N}^{2}}, \bar{G}_{11 N}^{*}=\frac{G_{11 N}^{*}}{\rho_{N} h_{N}^{3}}, \\
& \bar{G}_{11 p}^{*}=\frac{G_{11 p}^{*}}{\rho_{N} h_{N}^{3}}, \bar{N}_{x p}^{*}=\frac{N_{x p}^{*}}{A_{11 N}}, \bar{N}_{\theta p}^{*}=\frac{N_{\theta p}^{*}}{A_{11 N}}, \\
& \bar{M}_{x p}^{*}=\frac{M_{x p}^{*}}{A_{11 N} h_{N}}, \bar{M}_{\theta p}^{*}=\frac{M_{\theta p}^{*}}{A_{11 N} h_{N}}, \\
& \bar{\tau}_{0}^{S}=\frac{\tau_{0}^{S}}{A_{11 N}}, m_{0}=\frac{L}{R}=\frac{1}{\bar{R}}, m_{1}=\frac{L}{h_{N}}, \\
& m_{2}=\frac{h_{N}}{R}=\bar{h}_{N}, \bar{h}_{p}=\frac{h_{p}}{R}, m_{3}=\frac{I}{2 \rho_{N} h_{N}}, \\
& m_{4}=\frac{h_{p}}{h_{N}}, \mu=\frac{e_{0} a}{L}, \tau=t \sqrt{\frac{A_{11 N}}{2 \rho_{N} h_{N} L^{2}}}=\Omega t \text {, } \\
& \bar{\omega}=\frac{\omega}{\Omega}, \bar{K}_{w}=\frac{K_{w} L^{2}}{m_{3} A_{11 N}}, \bar{K}_{p}=\frac{K_{p}}{m_{3} A_{11 N}}, \\
& \bar{C}_{w}=\frac{C_{w}}{I},
\end{aligned}
$$

Respectively, strain and kinetic energies are obtained as follows:

$$
\begin{aligned}
& \pi=\frac{1}{2} \int_{0}^{L} \int_{0}^{2 \pi}\left\{\alpha_{1}\left(\frac{\partial \bar{u}}{\partial \xi}\right)^{2}+\alpha_{2} \frac{\partial \bar{u}}{\partial \xi} \frac{\partial \bar{v}}{\partial \theta}\right. \\
& +\alpha_{3} \bar{w} \frac{\partial \bar{u}}{\partial \xi}+\alpha_{4}\left(\frac{\partial \bar{v}}{\partial \theta}\right)^{2}+\alpha_{5} \bar{w}^{2}+\alpha_{6} \bar{w} \frac{\partial \bar{v}}{\partial \theta} \\
& +\alpha_{7}\left(\frac{\partial \bar{u}}{\partial \theta}\right)^{2}+\alpha_{8}\left(\frac{\partial \bar{v}}{\partial \xi}\right)^{2}+\alpha_{9} \frac{\partial \bar{v}}{\partial \xi} \frac{\partial \bar{u}}{\partial \theta}
\end{aligned}
$$

$$
\left.\begin{array}{l}
+\alpha_{10} \frac{\partial \bar{u}}{\partial \xi} \frac{\partial^{2} \bar{w}}{\partial \xi^{2}}+\alpha_{11} \frac{\partial \bar{u}}{\partial \xi} \frac{\partial^{2} \bar{w}}{\partial \theta^{2}}+\alpha_{12} \frac{\partial \bar{v}}{\partial \theta} \frac{\partial^{2} \bar{w}}{\partial \xi^{2}} \\
+\alpha_{13} \bar{w} \frac{\partial^{2} \bar{w}}{\partial \xi^{2}}+\alpha_{14} \frac{\partial \bar{v}}{\partial \theta} \frac{\partial^{2} \bar{w}}{\partial \theta^{2}}+\alpha_{15} \bar{w} \frac{\partial^{2} \bar{w}}{\partial \theta^{2}} \\
+\alpha_{16} \frac{\partial \bar{u}}{\partial \theta} \frac{\partial^{2} \bar{w}}{\partial \xi \partial \theta}+\alpha_{17} \frac{\partial \bar{v}}{\partial \xi} \frac{\partial^{2} \bar{w}}{\partial \xi \partial \theta}+\alpha_{18}\left(\frac{\partial^{2} \bar{w}}{\partial \xi^{2}}\right)^{2} \\
+\alpha_{19} \frac{\partial^{2} \bar{w}}{\partial \xi^{2}} \frac{\partial^{2} \bar{w}}{\partial \theta^{2}}+\alpha_{20}\left(\frac{\partial^{2} \bar{w}}{\partial \theta^{2}}\right)^{2}+\alpha_{21}\left(\frac{\partial^{2} \bar{w}}{\partial \xi \partial \theta}\right)^{2} \\
+\alpha_{22} \bar{w}+\alpha_{23} \frac{\partial \bar{u}}{\partial \xi}+\alpha_{24} \frac{\partial \bar{v}}{\partial \theta}+\alpha_{25} \frac{\partial^{2} \bar{w}}{\partial \xi^{2}} \\
+\alpha_{26} \frac{\partial^{2} \bar{w}}{\partial \theta^{2}}+\alpha_{27} \frac{\partial^{2} \bar{w}}{\partial \xi^{2}} \frac{\partial^{2} \bar{w}}{\partial \tau^{2}}+\alpha_{28} \frac{\partial^{2} \bar{w}}{\partial \theta^{2}} \frac{\partial^{2} \bar{w}}{\partial \tau^{2}} \\
+\alpha_{29} \frac{\partial \bar{u}}{\partial \xi} \frac{\partial^{2} \bar{w}}{\partial \tau^{2}}+\alpha_{30} \frac{\partial \bar{v}}{\partial \theta} \frac{\partial^{2} \bar{w}}{\partial \tau^{2}}+\alpha_{31} \bar{w} \frac{\partial^{2} \bar{w}}{\partial \tau^{2}} \\
\left.+\eta_{33} \bar{E}_{z p}^{2} h_{p}\right\} R d \theta d x, \\
T=\frac{1}{2} \int_{0}^{L} \int_{0}^{2 \pi}\left(\left(\frac{\partial \bar{u}}{\partial \tau}\right)^{2}+\left(\frac{\partial \bar{v}}{\partial \tau}\right)^{2}\right) R d \theta d x \\
+\left(\frac{\partial \bar{w}}{\partial \tau}\right)^{2}
\end{array}\right) R
$$

where coefficients of $\alpha_{k}(k=1 . .31)$ are introduced in Appendix 1.

Also the visco-pasternak effects are obtained as follows:

$W_{c}=-\frac{1}{2} \int_{0}^{L} \int_{0}^{2 \pi}\left\{\bar{C}_{w}\left(\frac{\partial \bar{w}}{\partial \tau}\right)^{2}\right\} R d \theta d x$

$W_{w p}$

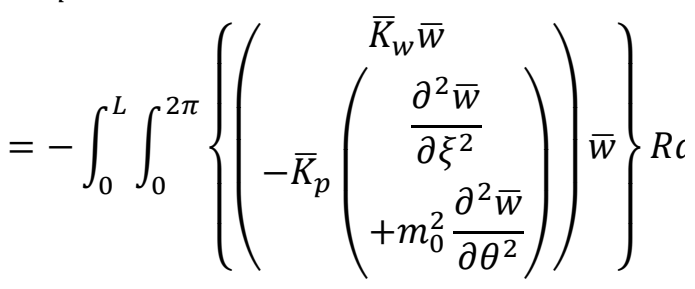

\subsection{Nonlocal Eringen shell theory}

According to Eringen [5, 6], for the piezoelectric cylindrical shell, the nonlocal constitutive relations for Eqs. (4) and (5) can be expressed

$$
\begin{aligned}
& \left\{\begin{array}{l}
\sigma_{x x N} \\
\sigma_{\theta \theta N} \\
\tau_{x \theta N}
\end{array}\right\}-\left(e_{0} a\right)^{2} \nabla^{2}\left\{\begin{array}{l}
\sigma_{x x N} \\
\sigma_{\theta \theta N} \\
\tau_{x \theta N}
\end{array}\right\} \\
& =\left[\begin{array}{ccc}
C_{11 N} & C_{12 N} & 0 \\
C_{21 N} & C_{22 N} & 0 \\
0 & 0 & C_{66 N}
\end{array}\right]\left\{\begin{array}{l}
\varepsilon_{x x} \\
\varepsilon_{\theta \theta} \\
\gamma_{x \theta}
\end{array}\right\},
\end{aligned}
$$

$$
\begin{aligned}
& \text { or }\left\{\sigma_{N}\right\}-\left(e_{0} a\right)^{2} \nabla^{2}\left\{\sigma_{N}\right\}=\left[C_{N}\right]\{\varepsilon\}, \\
& \left\{\begin{array}{l}
\sigma_{x x p} \\
\sigma_{\theta \theta p} \\
\tau_{x \theta p}
\end{array}\right\}-\left(e_{0} a\right)^{2} \nabla^{2}\left\{\begin{array}{l}
\sigma_{x x p} \\
\sigma_{\theta \theta p} \\
\tau_{x \theta p}
\end{array}\right\}
\end{aligned}
$$




$$
\begin{aligned}
& =\left[\begin{array}{ccc}
C_{11 p} & C_{12 p} & 0 \\
C_{21 p} & C_{22 p} & 0 \\
0 & 0 & C_{66 p}
\end{array}\right]\left\{\begin{array}{l}
\varepsilon_{x x} \\
\varepsilon_{\theta \theta} \\
\gamma_{x \theta}
\end{array}\right\} \\
& -\left[\begin{array}{ccc}
0 & 0 & e_{31 p} \\
0 & 0 & e_{32 p} \\
0 & 0 & 0
\end{array}\right]\left\{\begin{array}{l}
\bar{E}_{x p} \\
\bar{E}_{\theta p} \\
\bar{E}_{z p}
\end{array}\right\}, \\
& \text { or }\left\{\sigma_{p}\right\}-\left(e_{0} a\right)^{2} \nabla^{2}\left\{\sigma_{p}\right\} \\
& =\left[C_{p}\right]\{\varepsilon\}-\left[e_{p}\right]\left\{\bar{E}_{p}\right\},
\end{aligned}
$$

Also, the radial component of electric displacement $D_{z p}$ Eq. (9) can be presented as

$D_{z p}-\left(e_{0} a\right)^{2} \nabla^{2} D_{z p}=e_{31 p} \varepsilon_{x x}$

$+e_{32 p} \varepsilon_{\theta \theta}+\eta_{33 p} \bar{E}_{z p}$

where $\nabla^{2}=\partial^{2} / \partial x^{2}+\partial^{2} / \partial(R \theta)^{2}$ is the Laplace operator; $\left(e_{0} a\right)$ is the scale coefficient revealing the size effect on the response of nanostructures.

From Eqs. (35)-(37) and using Eqs. (25a, b) and ignoring of surface energy effects for $N_{x x}, N_{\theta \theta}, N_{x \theta}, M_{x x}, M_{\theta \theta}$ and $M_{x \theta}$ in Eqs. $(25 \mathrm{a}, \mathrm{b})$, we have

$$
\begin{aligned}
& N_{x x}-\left(e_{0} a\right)^{2} \nabla^{2} N_{x x}=A_{11} \varepsilon_{x x}^{0} \\
& +A_{12} \varepsilon_{\theta \theta}^{0}+B_{11} \kappa_{x x}+B_{12} \kappa_{\theta \theta}-N_{x p} \\
& N_{\theta \theta}-\left(e_{0} a\right)^{2} \nabla^{2} N_{\theta \theta}=A_{21} \varepsilon_{x x}^{0} \\
& +A_{22} \varepsilon_{\theta \theta}^{0}+B_{21} \kappa_{x x}+B_{22} \kappa_{\theta \theta}-N_{\theta p} \\
& N_{x \theta}-\left(e_{0} a\right)^{2} \nabla^{2} N_{x \theta}=A_{66} \gamma_{x \theta}^{0} \\
& +B_{66} \kappa_{x \theta} \\
& M_{x x}-\left(e_{0} a\right)^{2} \nabla^{2} M_{x x}=B_{11} \varepsilon_{x x}^{0} \\
& +B_{12} \varepsilon_{\theta \theta}^{0}+D_{11} \kappa_{x x}+D_{12} \kappa_{\theta \theta}-M_{x p}, \\
& M_{\theta \theta}-\left(e_{0} a\right)^{2} \nabla^{2} M_{\theta \theta}=B_{21} \varepsilon_{x x}^{0} \\
& +B_{22} \varepsilon_{\theta \theta}^{0}+D_{21} \kappa_{x x}+D_{22} \kappa_{\theta \theta}-M_{\theta p}, \\
& M_{x \theta}-\left(e_{0} a\right)^{2} \nabla^{2} M_{x \theta}=B_{66} \gamma_{x \theta}^{0}+D_{66} \kappa_{x \theta},
\end{aligned}
$$

Note that all relations of equations (25)-(27) with the elimination of all surface effect parameters are established for the Eringen nonlocal theory. Substituting Eqs. (38a)-(38f) into the governing equations (28a-c) and (29a-e) yields

$\delta u: \frac{\partial N_{x x}}{\partial x}+\frac{1}{R} \frac{\partial N_{x \theta}}{\partial \theta}$

$=\left(1-\left(e_{0} a\right)^{2} \nabla^{2}\right) I \frac{\partial^{2} u}{\partial t^{2}}$

$\delta v: \quad \frac{\partial N_{x \theta}}{\partial x}+\frac{1}{R} \frac{\partial N_{\theta \theta}}{\partial \theta}$

$=\left(1-\left(e_{0} a\right)^{2} \nabla^{2}\right) I \frac{\partial^{2} v}{\partial t^{2}}$ $\delta w: \frac{\partial^{2} M_{x x}}{\partial x^{2}}+\frac{2}{R} \frac{\partial^{2} M_{x \theta}}{\partial x \partial \theta}+\frac{1}{R^{2}} \frac{\partial^{2} M_{\theta \theta}}{\partial \theta^{2}}$

$-\frac{N_{\theta \theta}}{R}=\left(1-\left(e_{0} a\right)^{2} \nabla^{2}\right) \times$

$\left(\begin{array}{c}I \frac{\partial^{2} w}{\partial t^{2}}+C_{w} \frac{\partial w}{\partial t}+K_{w} w \\ -K_{p}\left(\frac{\partial^{2} w}{\partial x^{2}}+\frac{1}{R^{2}} \frac{\partial^{2} w}{\partial \theta^{2}}\right)\end{array}\right)$,

and boundary conditions are obtained as (29a)(29e). By ignoring of surface energy effects Eqs. (25c)-(25h) and substituting into Eqs. (39a)-(39c), we obtain the following equations of motion:

$$
\begin{aligned}
& A_{11} \frac{\partial^{2} u}{\partial x^{2}}+\frac{A_{66}}{R^{2}} \frac{\partial^{2} u}{\partial \theta^{2}}+\frac{\left(A_{12}+A_{66}\right)}{R} \frac{\partial^{2} v}{\partial x \partial \theta} \\
& +\frac{A_{12}}{R} \frac{\partial w}{\partial x}-B_{11} \frac{\partial^{3} w}{\partial x^{3}} \\
& +\frac{\left(-B_{12}-2 B_{66}\right)}{R^{2}} \frac{\partial^{3} w}{\partial x \partial \theta^{2}}+\frac{\partial}{\partial x}\left(-N_{x p}\right) \\
& =\left(1-\left(e_{0} a\right)^{2} \nabla^{2}\right) I \frac{\partial^{2} u}{\partial t^{2}}, \\
& \frac{A_{66}}{R} \frac{\partial^{2} u}{\partial \theta^{2}}+\frac{A_{21}}{R} \frac{\partial^{2} u}{\partial x \partial \theta}+A_{66} \frac{\partial^{2} v}{\partial x^{2}}+\frac{A_{22}}{R^{2}} \frac{\partial^{2} v}{\partial \theta^{2}} \\
& +\frac{A_{22}}{R^{2}} \frac{\partial w}{\partial \theta}-\frac{\left(B_{21}+2 B_{66}\right)}{R} \frac{\partial^{3} w}{\partial x^{2} \partial \theta} \\
& -\frac{B_{22}}{R^{3}} \frac{\partial^{3} w}{\partial \theta^{3}}-\frac{1}{R} \frac{\partial N_{\theta p}}{\partial \theta} \\
& =\left(1-\left(e_{0} a\right)^{2} \nabla^{2}\right) I \frac{\partial^{2} v}{\partial t^{2}}, \\
& -\frac{A_{21}}{R} \frac{\partial u}{\partial x}+B_{11} \frac{\partial^{3} u}{\partial x^{3}} \\
& +\frac{\left(B_{21}+2 B_{66}\right)}{R^{2}} \frac{\partial^{3} u}{\partial x \partial \theta^{2}}-\frac{A_{22}}{R^{2}} \frac{\partial v}{\partial \theta} \\
& +\frac{\left(B_{12}+2 B_{66}\right)}{R^{2}} \frac{\partial^{3} v}{\partial x^{2} \partial \theta}+\frac{B_{22}}{R^{3}} \frac{\partial^{3} v}{\partial \theta^{3}}-\frac{A_{22}}{R^{2}} w \\
& +\frac{\left(B_{12}+B_{21}\right)}{R} \frac{\partial^{2} w}{\partial x^{2}}+\frac{2 B_{22}}{R^{3}} \frac{\partial^{2} w}{\partial \theta^{2}} \\
& +D_{11} \frac{\partial^{4} w}{\partial x^{4}}-\frac{\left(D_{12}+D_{21}+4 D_{66}\right)}{R^{2}} \frac{\partial^{4} w}{\partial x^{2} \partial \theta^{2}} \\
& -\frac{D_{22}}{R^{4}} \frac{\partial^{4} w}{\partial \theta^{4}}+\frac{N_{\theta p}}{R}-\frac{\partial^{2} M_{x p}}{\partial x^{2}}-\frac{1}{R^{2}} \frac{\partial^{2} M_{\theta p}}{\partial \theta^{2}} \\
& =\left(1-\left(e_{0} a\right)^{2} \nabla^{2}\right) \times \\
& \left(\begin{array}{c}
I \frac{\partial^{2} w}{\partial t^{2}}+C_{w} \frac{\partial w}{\partial t}+K_{w} w \\
-K_{p}\left(\frac{\partial^{2} w}{\partial x^{2}}+\frac{1}{R^{2}} \frac{\partial^{2} w}{\partial \theta^{2}}\right)
\end{array}\right)
\end{aligned}
$$

And by introducing the dimensionless parameters (30), Eqs. (40a)-(40c) can be expressed in the dimensionless form as 


$$
\begin{aligned}
& \beta_{1} \frac{\partial^{2} \bar{u}}{\partial \xi^{2}}+\beta_{2} \frac{\partial^{2} \bar{u}}{\partial \theta^{2}}+\beta_{3} \frac{\partial^{2} \bar{v}}{\partial \xi \partial \theta}+\beta_{4} \frac{\partial \bar{w}}{\partial \xi} \\
& +\beta_{5} \frac{\partial^{3} \bar{w}}{\partial \xi^{3}}+\beta_{6} \frac{\partial^{3} \bar{w}}{\partial \xi \partial \theta^{2}}=\left(1-\mu^{2} \bar{\nabla}^{2}\right) \frac{\partial^{2} \bar{u}}{\partial \tau^{2}}, \\
& \beta_{7} \frac{\partial^{2} \bar{u}}{\partial \theta^{2}}+\beta_{8} \frac{\partial^{2} \bar{u}}{\partial \xi \partial \theta}+\beta_{9} \frac{\partial^{2} \bar{v}}{\partial \xi^{2}}+\beta_{10} \frac{\partial^{2} \bar{v}}{\partial \theta^{2}} \\
& +\beta_{11} \frac{\partial \bar{w}}{\partial \theta}+\beta_{12} \frac{\partial^{3} \bar{w}}{\partial \xi^{2} \partial \theta}+\beta_{13} \frac{\partial^{3} \bar{w}}{\partial \theta^{3}} \\
& =\left(1-\mu^{2} \bar{\nabla}^{2}\right) \frac{\partial^{2} \bar{v}}{\partial \tau^{2}} \\
& \beta_{14} \frac{\partial \bar{u}}{\partial \xi}+\beta_{15} \frac{\partial^{3} \bar{u}}{\partial \xi^{3}}+\beta_{16} \frac{\partial^{3} \bar{u}}{\partial \xi \partial \theta^{2}}+\beta_{17} \frac{\partial \bar{v}}{\partial \theta} \\
& +\beta_{18} \frac{\partial^{3} \bar{v}}{\partial \xi^{2} \partial \theta}+\beta_{19} \frac{\partial^{3} \bar{v}}{\partial \theta^{3}}+\beta_{20} \bar{w} \\
& +\beta_{21} \frac{\partial^{2} \bar{w}}{\partial \xi^{2}}+\beta_{22} \frac{\partial^{2} \bar{w}}{\partial \theta^{2}}+\beta_{23} \frac{\partial^{4} \bar{w}}{\partial \xi^{4}} \\
& +\beta_{24} \frac{\partial^{4} \bar{w}}{\partial \xi^{2} \partial \theta^{2}}+\beta_{25} \frac{\partial^{4} \bar{w}}{\partial \theta^{4}}+\beta_{26} \\
& =\left(1-\mu^{2} \bar{\nabla}^{2}\right) \times \\
& \left(\begin{array}{c}
\frac{\partial^{2} \bar{w}}{\partial \tau^{2}}+\bar{C}_{w} \frac{\partial \bar{w}}{\partial \tau}+\bar{K}_{w} \bar{w} \\
-\bar{K}_{p}\left(\frac{\partial^{2} \bar{w}}{\partial \xi^{2}}+m_{0}^{2} \frac{\partial^{2} \bar{w}}{\partial \theta^{2}}\right)
\end{array}\right)
\end{aligned}
$$

where $\bar{\nabla}^{2}=\partial^{2} / \partial \xi^{2}+m_{0}^{2} \partial^{2} / \partial \theta^{2}$ and also coefficients of $\beta_{k}(k=1 . .26)$ are introduced in Appendix 2. Then, using Eqs. (25a, b) and ignoring of surface energy effects for $N_{x x}, N_{\theta \theta}, N_{x \theta}, M_{x x}$, $M_{\theta \theta}$, and $M_{x \theta}$ and substitution into boundary conditions Eqs. (29a)-(29e) and dimensionless parameters (30), the boundary conditions can be expressed in the dimensionless form as

$\delta \bar{u}=0,\left.\left(\begin{array}{c}\bar{\beta}_{1} \frac{\partial \bar{u}}{\partial \xi}+\bar{\beta}_{2} \frac{\partial \bar{v}}{\partial \theta}+\bar{\beta}_{3} \bar{w} \\ +\bar{\beta}_{4} \frac{\partial^{2} \bar{w}}{\partial \xi^{2}}+\bar{\beta}_{5} \frac{\partial^{2} \bar{w}}{\partial \theta^{2}}+\bar{\beta}_{6}\end{array}\right) x\right|_{0} ^{1} n_{x}$

$$
+\left.\left(\bar{\beta}_{7} \frac{\partial \bar{u}}{\partial \theta}+\bar{\beta}_{8} \frac{\partial \bar{v}}{\partial \xi}+\bar{\beta}_{9} \frac{\partial^{2} \bar{w}}{\partial \xi \partial \theta}\right) \theta\right|_{0} ^{2 \pi}=0,
$$$$
\delta \bar{v}=0,\left.\left(\begin{array}{c}
\bar{\beta}_{10} \frac{\partial \bar{u}}{\partial \theta}+\bar{\beta}_{11} \frac{\partial \bar{v}}{\partial \xi} \\
+\bar{\beta}_{12} \frac{\partial^{2} \bar{w}}{\partial \xi \partial \theta}
\end{array}\right) x\right|_{0} ^{1} n_{x}
$$$$
+\left.\left(\begin{array}{c}
\bar{\beta}_{13} \frac{\partial \bar{u}}{\partial \xi}+\bar{\beta}_{14} \frac{\partial \bar{v}}{\partial \theta}+\bar{\beta}_{15} \bar{w} \\
+\bar{\beta}_{16} \frac{\partial^{2} \bar{w}}{\partial \xi^{2}}+\bar{\beta}_{17} \frac{\partial^{2} \bar{w}}{\partial \theta^{2}}+\bar{\beta}_{18}
\end{array}\right) \theta\right|_{0} ^{2 \pi}=0,
$$

$\delta \bar{w}=0$,

$$
\begin{gathered}
\left.\left(\begin{array}{c}
\bar{\beta}_{19} \frac{\partial^{2} \bar{u}}{\partial \xi^{2}}+\bar{\beta}_{20} \frac{\partial^{2} \bar{u}}{\partial \theta^{2}}+\bar{\beta}_{21} \frac{\partial^{2} \bar{v}}{\partial \xi \partial \theta} \\
+\bar{\beta}_{22} \frac{\partial \bar{w}}{\partial \xi}+\bar{\beta}_{23} \frac{\partial^{3} \bar{w}}{\partial \xi^{3}} \\
+\bar{\beta}_{24} \frac{\partial^{3} \bar{w}}{\partial \xi \partial \theta^{2}}
\end{array}\right) x\right|_{0} ^{1} n_{x} \\
+\left.\left(\begin{array}{c}
\bar{\beta}_{25} \frac{\partial^{2} \bar{u}}{\partial \xi \partial \theta}+\bar{\beta}_{26} \frac{\partial^{2} \bar{v}}{\partial \xi^{2}}+\bar{\beta}_{27} \frac{\partial^{2} \bar{v}}{\partial \theta^{2}} \\
+\bar{\beta}_{28} \frac{\partial \bar{w}}{\partial \theta}+\bar{\beta}_{29} \frac{\partial^{3} \bar{w}}{\partial \theta^{3}} \\
+\bar{\beta}_{30} \frac{\partial^{3} \bar{w}}{\partial \xi^{2} \partial \theta}
\end{array}\right) \theta\right|_{0} ^{2 \pi}=0
\end{gathered}
$$

$$
\frac{\partial \bar{w}}{\partial \xi}=0 \text {, }
$$$$
\left.\left(\begin{array}{c}
\bar{\beta}_{31} \frac{\partial \bar{u}}{\partial \xi}+\bar{\beta}_{32} \frac{\partial \bar{v}}{\partial \theta}+\bar{\beta}_{33} \bar{w} \\
+\bar{\beta}_{34} \frac{\partial^{2} \bar{w}}{\partial \xi^{2}}+\bar{\beta}_{35} \frac{\partial^{2} \bar{w}}{\partial \theta^{2}}+\bar{\beta}_{36}
\end{array}\right) x\right|_{0} ^{1}
$$$$
+\left.\left(\bar{\beta}_{37} \frac{\partial \bar{u}}{\partial \theta}+\bar{\beta}_{38} \frac{\partial \bar{v}}{\partial \xi}+\bar{\beta}_{39} \frac{\partial^{2} \bar{w}}{\partial \xi \partial \theta}\right) \theta\right|_{0} ^{2 \pi}=0,
$$

$\frac{\partial \bar{w}}{\partial \theta}=0$,

$\left.\left(\bar{\beta}_{40} \frac{\partial \bar{u}}{\partial \theta}+\bar{\beta}_{41} \frac{\partial \bar{v}}{\partial \xi}+\bar{\beta}_{42} \frac{\partial^{2} \bar{w}}{\partial \xi \partial \theta}\right) x\right|_{0} ^{1} n_{x}$

$+\left.\left(\begin{array}{c}\bar{\beta}_{43} \frac{\partial \bar{u}}{\partial \xi}+\bar{\beta}_{44} \frac{\partial \bar{v}}{\partial \theta}+\bar{\beta}_{45} \bar{w} \\ +\bar{\beta}_{46} \frac{\partial^{2} \bar{w}}{\partial \xi^{2}}+\bar{\beta}_{47} \frac{\partial^{2} \bar{w}}{\partial \theta^{2}}+\bar{\beta}_{48}\end{array}\right) \theta\right|_{0} ^{2 \pi}=0$,

where coefficients of $\bar{\beta}_{k}(k=1 . .48)$ are introduced in Appendix 3.

\subsection{Solution procedure}

In the assumed mode method, displacement and shear deformation are written in terms of generalized coordinate and mode function as follows [38]:

$$
\begin{aligned}
& u(x, \theta, t)= \\
& \sum_{m=1}^{M_{1}} \sum_{j=1}^{N}\left[\begin{array}{l}
u_{m, j, c}(\tau) \cos (j \theta) \\
+u_{m, j, s}(\tau) \sin (j \theta)
\end{array}\right] \chi_{m j}(\xi) \\
& +\sum_{\substack{m=1 \\
M_{2}+M_{1} \times N}}^{M_{2}} u_{m, 0}(\tau) \chi_{m 0}(\xi)= \\
& \sum_{i=1} u_{i}(\tau) \chi_{i}(\xi) \vartheta_{i}(\theta)
\end{aligned}
$$




$$
\begin{aligned}
& v(x, \theta, t)= \\
& \sum_{m=1}^{M_{1}} \sum_{j=1}^{N}\left[\begin{array}{c}
v_{m, j, c}(\tau) \sin (j \theta) \\
+v_{m, j, s}(\tau) \cos (j \theta)
\end{array}\right] \phi_{m j}(\xi) \\
& +\sum_{m=1}^{M_{2}} v_{m, 0}(\tau) \phi_{m 0}(\xi)= \\
& \sum_{r=1}^{M_{2}+M_{1} \times N} v_{r}(\tau) \phi_{r}(\xi) \alpha_{r}(\theta) \\
& w(x, \theta, t)= \\
& \sum_{m=1}^{M_{1}} \sum_{j=1}^{N}\left[\begin{array}{c}
w_{m, j, c}(\tau) \cos (j \theta) \\
+w_{m, j, s}(\tau) \sin (j \theta)
\end{array}\right] \beta_{m j}(\xi) \\
& +\sum_{m=1}^{M_{2}} w_{m, 0}(\tau) \beta_{m 0}(\xi)= \\
& \sum_{s=1}^{M_{2}+M_{1} \times N} \\
& \sum_{s}(\tau) \beta_{s}(\xi) \psi_{s}(\theta)
\end{aligned}
$$

where $\chi_{i}(\xi), \phi_{r}(\xi)$ and $\beta_{s}(\xi)$ are modal functions which satisfy the required geometric boundary conditions. $u_{i}(\tau), v_{r}(\tau)$ and $w_{s}(\tau)$ are generalized coordinates. In the present work, the Euler Bernoulli bending mode shapes are used to solve the classical theory of shell as $\beta_{S}(\xi)$ and also the mode shape of the rod is used for $\chi_{i}(\xi)$ and $\phi_{r}(\xi)$ modal functions which satisfy the required geometric boundary conditions in all shell theories for these two directions.

Substituting Eqs. (43) into Eqs. (31)-(34) and using Euler-Lagrange method the following reducedorder model of the system is obtained.

$$
\begin{aligned}
& {\left[(M)_{u L}^{u}\right]\{\ddot{\ddot{u}}\}+\left[(M)_{u L}^{w}\right]\{\ddot{\bar{w}}\}+\left[(K)_{u L}^{u}\right]\{\bar{u}\}} \\
& +\left[(K)_{u L}^{v}\right]\{\bar{v}\}+\left[(K)_{u L}^{w}\right]\{\bar{w}\}=\bar{F}_{u p L} \\
& {\left[(M)_{v L}^{v}\right]\{\ddot{\bar{v}}\}+\left[(M)_{v L}^{w}\right]\{\ddot{\bar{w}}\}+\left[(K)_{v L}^{u}\right]\{\bar{u}\}} \\
& +\left[(K)_{v L}^{v}\right]\{\bar{v}\}+\left[(K)_{v L}^{w}\right]\{\bar{w}\}=\bar{F}_{v p L} \\
& {\left[(M)_{w L}^{w}\right]\{\ddot{\bar{w}}\}+\left[(C)_{w L}^{w}\right]\{\dot{\bar{w}}\}+\left[(K)_{w L}^{u}\right]\{\bar{u}\}} \\
& +\left[(K)_{w L}^{v}\right]\{\bar{v}\}+\left[(K)_{w L}^{w}\right]\{\bar{w}\}=\bar{F}_{w p L},
\end{aligned}
$$

where $\left[(M)_{q L}^{r}\right],\left[(C)_{w}^{w}\right]$ and $\left[(K)_{q}^{r}\right]$ are mass, damping and stiffness matrices, respectively, in directions of $u, v$ and $w(r, q=u, v, w)$. Also, $\bar{F}_{u p L}, \quad \bar{F}_{v p L}$ and $\bar{F}_{w p L}$ are applied loads by piezoelectric voltage and surface stress. For free vibration of piezoelectric nanoshell $\bar{F}$ are zero, and as a result $\left(\bar{F}_{u p L}=\bar{F}_{v p L}=\bar{F}_{w p L}=0\right)$. All coefficients of mass and stiffness matrixes Eqs. 44(a)-(c) are presented in Appendix 4. Also for
Hamilton principle, substituting Eqs. (43) into Eqs. (41)-(42) results in the following reduced-order model of the system:

$$
\begin{aligned}
& {\left[(M)_{u H}^{u}+\left(M_{b c}\right)_{u H}^{u}\right]\{\ddot{\bar{u}}\}} \\
& +\left[(K)_{u H}^{u}+\left(K_{b c}\right)_{u H}^{u}\right]\{\bar{u}\} \\
& +\left[(K)_{u H}^{v}+\left(K_{b c}\right)_{u H}^{v}\right]\{\bar{v}\} \\
& +\left[(K)_{u H}^{w}+\left(K_{b c}\right)_{u H}^{w}\right]\{\bar{w}\}+\bar{F}_{u p H}=0, \\
& {\left[(M)_{v H}^{v}\right]\{\ddot{\bar{v}}\}+\left[(K)_{v H}^{u}+\left(K_{b c}\right)_{v H}^{u}\right]\{\bar{u}\}} \\
& +\left[(K)_{v H}^{v}+\left(K_{b c}\right)_{v H}^{v}\right]\{\bar{v}\}+\left[(K)_{v H}^{w}\right]\{\bar{w}\} \\
& +\bar{F}_{v p H}=0, \\
& {\left[(M)_{w H}^{w}\right]\{\ddot{\bar{w}}\}+\left[(C)_{w H}^{w}\right]\{\dot{\bar{w}}\}} \\
& +\left[(K)_{w H}^{u}+\left(K_{b c}\right)_{w H}^{u}\right]\{\bar{u}\} \\
& +\left[(K)_{w H}^{v}+\left(K_{b c}\right)_{w H}^{v}\right]\{\bar{v}\} \\
& +\left[(K)_{w H}^{w}+\left(K_{b c}\right)_{w H}^{w}\right]\{\bar{w}\}+\bar{F}_{w p H}=0,
\end{aligned}
$$

where $\left[(M)_{q H}^{r}\right],\left[(C)_{w H}^{w}\right]$ and $\left[(K)_{q H}^{r}\right]$ are mass, damping and stiffness matrices, respectively, in directions of $u, v$ and $w(r, q=u, v, w)$. Also, $\bar{F}_{u p H}, \quad \bar{F}_{v p H}$ and $\bar{F}_{w p H}$ are applied loads by piezoelectric voltage and surface stress and for free vibration of piezoelectric nanoshell $\bar{F}$ are zero, as a result $\left(\bar{F}_{u p}=\bar{F}_{v p}=\bar{F}_{w p}=0\right)$. All coefficients of mass and stiffness matrixes Eqs. 45(a)-(c) are presented in Appendix 5.

Natural frequencies and mode shapes can be obtained from solving following eigenvalue equation:

$\left[[K]-\omega_{m n}^{2}[M]\right]\left\{u_{m n} v_{m n} w_{m n}\right\}^{T}=0$,

\section{Numerical results and Discussions}

In this section, at first, convergence study of a piezoelectric cylindrical nano-shell with arbitrary boundary conditions is investigated for GurtinMurdoch surface/interface theory and Eringen nonlocal theory. Then the surface energy effects using the Gurtin-Murdoch surface/interface theory on the free vibration analysis of a piezoelectric cylindrical nano-shell with arbitrary boundary conditions is investigated and at the end, the natural frequency analysis of a simply supported piezoelectric cylindrical nano-shell is presented using the Eringen nonlocal theory. In order to simplify the presentation, $\mathrm{CC}, \mathrm{SS}, \mathrm{CS}$ and $\mathrm{CF}$ represent clamped edges, simply supported edges, clamped-simply supported edges and clamped-free edges, respectively and also for simplification of surface effect is represented SE. The non- 
homogeneous nano-shell considered in the following examples is composed of stainless steel and nickel and the nonhomogeneous distribution of properties in the thickness direction is varied according to the volume fraction power-law function. The material properties for nanoshell (stainless steel and nickel) and also the piezoelectric layer (PZT-4 material) are shown in Table 1 and Table 2, respectively [42].

Table 1 Properties of stainless steel and nickel [42]

\begin{tabular}{|c|c|c|c|c|c|}
\hline \multicolumn{3}{|l|}{ Stainless steel } & \multicolumn{3}{l|}{ Nichel } \\
\hline $\boldsymbol{E}_{\boldsymbol{B}}(\boldsymbol{G P C}$ & $v_{B}$ & $\rho_{B}\left(\mathrm{~kg} \mathrm{~m}^{-}\right.$ & $E_{T}(G P c$ & $v_{T}$ & $\rho_{T}\left(\mathrm{~kg} \mathrm{~m}^{-}\right.$ \\
\hline $\mathbf{2 0 8}$ & 0.381 & 8166 & 205 & 0.31 & 8900 \\
\hline
\end{tabular}

Table 2 Properties of PZT-4 [42]

\begin{tabular}{|c|c|c|c|c|c|}
\hline $\boldsymbol{E}_{\boldsymbol{p}}(\boldsymbol{G H}$ & $\boldsymbol{v}_{\boldsymbol{p}}$ & $\boldsymbol{e}_{\mathbf{3 1} \boldsymbol{p}}(\boldsymbol{C})$ & $\boldsymbol{e}_{\mathbf{3 2 p}}(\boldsymbol{C})$ & $\boldsymbol{\eta}_{\mathbf{3 3 p}}\left(\mathbf{1 0}^{-\mathbf{1}}\right.$ & $\boldsymbol{\rho}_{\boldsymbol{p}}(\boldsymbol{k g} \boldsymbol{\eta}$ \\
\hline $\mathbf{9 5}$ & 0.3 & -5.2 & -5.2 & 560 & 7500 \\
\hline
\end{tabular}

Also, the material property of surface effects and geometrical parameters used in all following results are shown in Table 3.

Table 3 The material property of surface effects and geometrical parameters

\begin{tabular}{|c|c|c|c|c|c|}
\hline$R(m)$ & $L / R$ & $\boldsymbol{h}_{N} / \boldsymbol{R}$ & $h_{p} / R$ & $\lambda^{s_{1}}(\mathrm{~N} /$ & $\mu^{s_{1}}(N)$ \\
\hline $1 \times 10$ & 10 & 0.05 & 0.03 & 4.448 & 2.774 \\
\hline $\begin{array}{c}\tau_{0}^{s_{1}} \\
(\boldsymbol{N} / m)\end{array}$ & $\rho^{s_{1}}(k g$ & $V_{p}(V)$ & $\lambda^{S_{2}}(N / \imath$ & $\mu^{s_{2}}(N /$ & $\tau_{0}^{S_{2}}(N)$ \\
\hline 0.6 & $3.17 \times$ & $1 \times 10^{-}$ & 4.448 & 2.774 & 0.6 \\
\hline$e_{31 p}^{s}(C$ & $e_{32 p}^{S}(C$ & $\rho^{S_{2}}(\mathrm{~kg}$ & $C_{w}(N . s$ & $K_{w}(N)$ & $\begin{array}{l}K_{p}( \\
N)\end{array}$ \\
\hline$-3 \times 1$ & $-3 \times 10$ & $5.61 \times$ & $\begin{array}{l}3 \\
\times 10^{-7}\end{array}$ & $\begin{array}{l}1 \\
\times 10^{20}\end{array}$ & $\begin{array}{l}1 \\
\times 10^{-2}\end{array}$ \\
\hline
\end{tabular}

Of course, the geometrical parameters can be varying according to the type of problem. In this paper, the results are presented in dimensionless form and thus the results are not limited to a particular type of matter. The data presented in the form of sample data to approximate the numbers used in the actual range.

\subsection{Convergence and comparison studies}

The method proposed in this paper is validated by comparing the present numerical results with previously published in the literature. If we neglect the piezoelectric, visco-Pasternak and surface effects, the present model can be reduced to the macroscopic cylindrical shell model. The dimensionless natural frequencies $\left(\omega_{n}=\right.$ $\left.\Omega R \sqrt{\left(1-v^{2}\right) \rho / E}\right)$ of present work are compared with macroscopic cylindrical shell which previously given by Loy et al. [26] that is shown in Table 4 for the three classical boundary conditions. The parameters used in this example are: $m=1$, $L / R=20, h_{N} / R=0.01$, and $v=0.3$. It can be observed from Table 4 that the present results agree very well with the reference solutions, which indicates that the method presented in this paper is suitable and of high accuracy for free vibration analysis of cylindrical shells with classical boundary conditions. The slight differences in the results may be attributed to the different shell theories and solution approaches adopted in the literature and in this paper.

Table 4 Comparison of dimensionless natural frequencies for SS, SC and CC boundary conditions for a homogeneous cylindrical shells

\begin{tabular}{|l|l|l|l|l|l|l|}
\hline $\boldsymbol{n}$ & SS & \multicolumn{2}{l|}{ CS } & \multicolumn{2}{l|}{ CC } \\
\hline & $\begin{array}{l}\text { Prese } \\
\text { nt }\end{array}$ & $\begin{array}{l}\text { Loy } \\
\text { et al. } \\
(1997 \\
)\end{array}$ & $\begin{array}{l}\text { Prese } \\
\text { nt }\end{array}$ & $\begin{array}{l}\text { Loy } \\
\text { et al. } \\
(1997 \\
)\end{array}$ & $\begin{array}{l}\text { Prese } \\
\text { nt }\end{array}$ & $\begin{array}{l}\text { Loy } \\
\text { et al. } \\
(1997 \\
)\end{array}$ \\
\hline 1 & 0.016 & 0.016 & 0.023 & 0.023 & 0.034 & 0.032 \\
& 101 & 101 & 299 & 974 & 074 & 885 \\
\hline 2 & 0.005 & 0.009 & 0.010 & 0.011 & 0.014 & 0.013 \\
& 225 & 382 & 963 & 225 & 202 & 932 \\
\hline 3 & 0.021 & 0.022 & 0.018 & 0.022 & 0.018 & 0.022 \\
& 753 & 105 & 553 & 310 & 713 & 672 \\
\hline 4 & 0.034 & 0.042 & 0.036 & 0.042 & 0.041 & 0.042 \\
& 303 & 095 & 300 & 139 & 386 & 208 \\
\hline
\end{tabular}

Also, in all wave numbers shown, the natural frequency of the $\mathrm{CC}$ is greater, and the natural frequency of the SS is lower than the rest. The only significant difference is related to the state of SS in modes $n=2$ and $n=4$. By removing the equation from the dimensionless state and by referring to the analytical solution given by Rao SS [45], the solution obtained by the present paper, compared to reference Loy et al. [26], is much closer to the 
solution presented in reference Rao SS [45], as a result the lower frequency found in this paper is correct. As an example of the Rao SS [45], the natural frequencies of transverse vibration of a circular cylindrical shell simply supported at $x=0$ and $x=l$ for the following data: $E=30 \times$ $10^{6} \mathrm{psi}, \quad v=0.3, \rho=7.324 \times 10^{-1} 1 \mathrm{~b}-$ $\mathrm{sec}^{2} / \mathrm{in}^{4}, R=10 \mathrm{in}, l=40 \mathrm{in}$ and $h=0.1 \mathrm{in}$ for the $(m, n)=(1,2),(1,3)$ and $(3,2)$ are $2,375.8223$, $1,321.9526$ and 10,086.031, respectively and for present work with the same data as given reference Rao SS [45] and for the $(m, n)=(1,2),(1,3)$ and $(3,2)$ are $2,394.635,1,343.182$ and 10,211.415, respectively. The slight differences in the results may be attributed to the different shell theories and solution approaches adopted in the literature and in this paper.

Table 5 show a complete convergence of the natural frequency parameter, $\omega_{n}$, for SS, CS, CC and $\mathrm{CF}$ piezoelectric nanoshell for $N=2,4,6$ considering with the Gurtin-Murdoch surface/interface theory and the material and geometrical parameters of Tables (1-3). It is observed that for all boundary conditions as the number of polynomial terms, $N$, is increased, the value of the frequency parameter, $\omega_{n}$, converges rapidly. With considering of the two succesasing values of $N$, it is shows that as $N$ increases, the percentage difference between the successive frequency approximations decreases. Thus the error as shown above is less 1 per cent, which is well within the limits of engineering tolerance. The minimum frequency in this case is associated with the circumferential wave number $n=2$. This assertion is valid for the entire range of shell parameters and for all type of boundary conditions.

Table 5 Convergence of dimensionless undamped natural frequencies $\omega_{n}=\bar{\Omega}_{n} R / L$ of the SS, SC,

$\mathrm{CC}$ and CF piezoelectric cylindrical shells

\begin{tabular}{|r|l|l|l|l|l|l|}
\hline & \multicolumn{3}{|c|}{ SS } & \multicolumn{3}{c|}{ CS } \\
\hline $\boldsymbol{n}$ & $\begin{array}{l}N \\
=2\end{array}$ & $\begin{array}{l}N \\
=4\end{array}$ & $\begin{array}{l}N \\
=6\end{array}$ & \multicolumn{1}{|c|}{$=2$} & \multicolumn{1}{|c|}{$=4$} & $\begin{array}{l}N \\
=6\end{array}$ \\
\hline 0 & 0.059 & 0.059 & 0.059 & 0.059 & 0.059 & 0.059 \\
& 839 & 839 & 839 & 839 & 839 & 839 \\
\hline 1 & 0.021 & 0.021 & 0.021 & 0.029 & 0.024 & 0.022 \\
& 835 & 835 & 835 & 098 & 138 & 793 \\
\hline
\end{tabular}

\begin{tabular}{|c|l|l|l|l|l|l|}
\hline 2 & 0.025 & 0.025 & 0.025 & 0.027 & 0.026 & 0.025 \\
& 465 & 465 & 465 & 229 & 294 & 910 \\
\hline 3 & 0.059 & 0.059 & 0.059 & 0.059 & 0.059 & 0.059 \\
& 553 & 553 & 554 & 553 & 442 & 408 \\
\hline & \multicolumn{3}{|c|}{ CC } & \multicolumn{3}{|c|}{ CF } \\
\hline $\boldsymbol{n}$ & $N$ & $N$ & $N$ & $N$ & $N$ & $N$ \\
\cline { 2 - 7 }$=2$ & $=4$ & $=6$ & $=2$ & $=4$ & $=6$ \\
\hline 0 & 0.059 & 0.059 & 0.059 & 0.029 & 0.029 & 0.029 \\
& 839 & 839 & 839 & 919 & 919 & 919 \\
\hline 1 & 0.047 & 0.027 & 0.024 & 0.012 & 0.008 & 0.007 \\
& 385 & 307 & 021 & 998 & 620 & 387 \\
\hline 2 & 0.061 & 0.028 & 0.026 & 0.026 & 0.024 & 0.024 \\
& 296 & 399 & 886 & 962 & 242 & 108 \\
\hline 3 & 0.085 & 0.059 & 0.059 & 0.060 & 0.059 & 0.059 \\
& 927 & 707 & 517 & 695 & 190 & 171 \\
\hline
\end{tabular}

As can be seen from Table 5, in all wave numbers shown, the natural frequency of the $\mathrm{CC}$ is greater, and the natural frequency of the CF is lower than the rest. Also, a convergence and accuracy study of the natural frequency $\omega_{n}$ of the SS piezoelectric nanoshells for Eringen nonlocal theory with the material and geometrical parameters of Tables (13 ) is presented in Table 6 with varying total numbers of nodes $N$ and for various circumferential wave numbers $n$.

Table 6 Convergence of dimensionless undamped natural frequencies $\omega_{n}=\bar{\Omega}_{n} R / L$ of the Eringen nonlocal theory for SS piezoelectric cylindrical

shells

\begin{tabular}{|l|l|l|l|l|}
\hline & \multicolumn{2}{|c|}{$\boldsymbol{\mu}=\mathbf{0}$} & \multicolumn{1}{c|}{$\boldsymbol{\mu}=\mathbf{0 . 0 2}$} \\
\hline $\boldsymbol{n}$ & $N=1$ & $N=2$ & $N=1$ & $N=2$ \\
\hline 1 & 0.567012 & 0.543459 & 0.554995 & 0.531641 \\
\hline 2 & 1.064967 & 1.060514 & 0.987180 & 0.982913 \\
\hline 3 & 1.577035 & 1.575583 & 1.350387 & 1.349085 \\
\hline 4 & 2.093065 & 2.092441 & 1.632448 & 1.631933 \\
\hline & \multicolumn{2}{|c|}{$\mu=0.05$} & \multicolumn{2}{c|}{$\mu=0.1$} \\
\hline $\boldsymbol{n}$ & $N=1$ & $N=2$ & $N=1$ & $N=2$ \\
\hline 1 & 0.502426 & 0.480069 & 0.391778 & 0.372328 \\
\hline 2 & 0.748597 & 0.744961 & 0.471781 & 0.469163 \\
\hline 3 & 0.871548 & 0.870564 & 0.496306 & 0.495676 \\
\hline 4 & 0.933748 & 0.933389 & 0.506176 & 0.505961 \\
\hline
\end{tabular}


Note that the nonlocal parameter $\mu=0$ corresponds to the piezoelectric cylindrical nanoshell without the nonlocal effect. As can be seen from Table 5, the dimensionless natural frequency of SS piezoelectric nanoshells decreases with increase of nonlocal parameter $\mu$. The reason is that a higher nonlocal parameter $\mu$ leads to a decrease in the nanoshell stiffness, and cause to lower natural frequencies of nanoshell, showing the softening effect observed by others (see Ke [46]). Also, for all nonlocal parameter $\mu$, the natural frequencies decrease with increase of the node number $N$ and increase with increase of the circumferential wave number $n$. As a result, the convergence mode number for both cases of the paper results (Table 5-6), i.e., Gurtin-Murdoch surface/interface and Eringen nonlocal theories is $n=2$ and $=2$. Some other studies in Applied Mechanics can be checked in [43] and [44].

\subsection{Parametric study}

The convergence and comparison study of the present work was verified in the previous subsection. In this subsection, we will study the effect of important parameters of cylindrical piezoelectric nanoshell on vibration behavior of this system.

In this subsection, first, the surface energy effects using the Gurtin-Murdoch surface/interface theory on the free vibration analysis of a piezoelectric cylindrical nanoshell with arbitrary boundary conditions is investigated and then, the natural frequency analysis of a simply supported (SS) piezoelectric cylindrical nanoshell is presented using the Eringen nonlocal theory.

Figure 2 (a, b) illustrates the effect of dimensionless stiffness coefficient of Winkler foundation $\bar{K}_{w}$ on dimensionless undamped natural frequencies $\left(\omega_{n}=\bar{\Omega}_{n} R / L\right)$ of the piezoelectric nano-shell. It can be seen that for all boundary conditions with and without surface energy effects, with the increase of the nanoshell stiffness coefficient, the fundamental frequency increases. As can be seen from Figure $2(a, b)$, the natural frequency of the $\mathrm{CC}$ is greater, and the natural frequency of the $\mathrm{CF}$ is lower than the rest. It is quite clear that considering the effects of the surface energy will lead to a significant decrease in the natural frequency of the nanoshell.

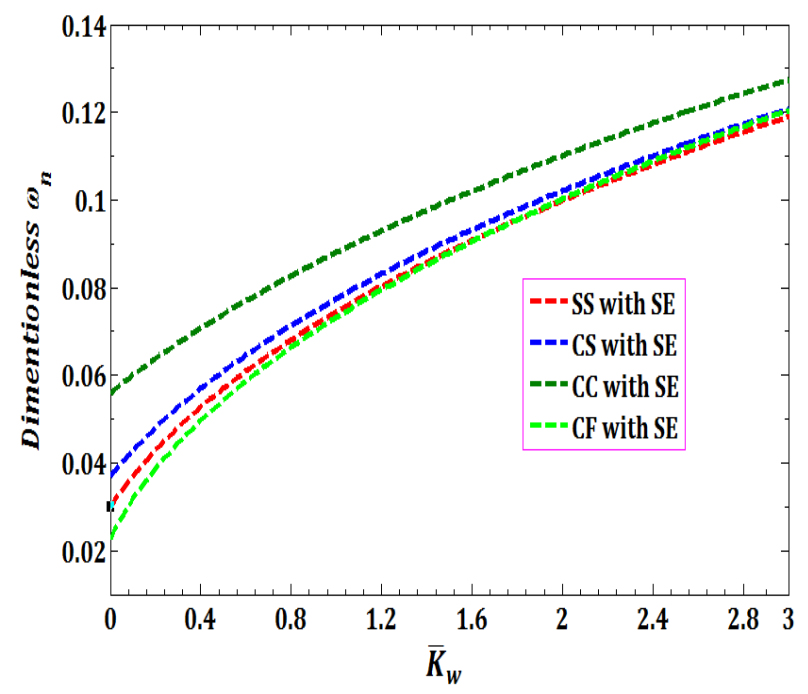

(a)

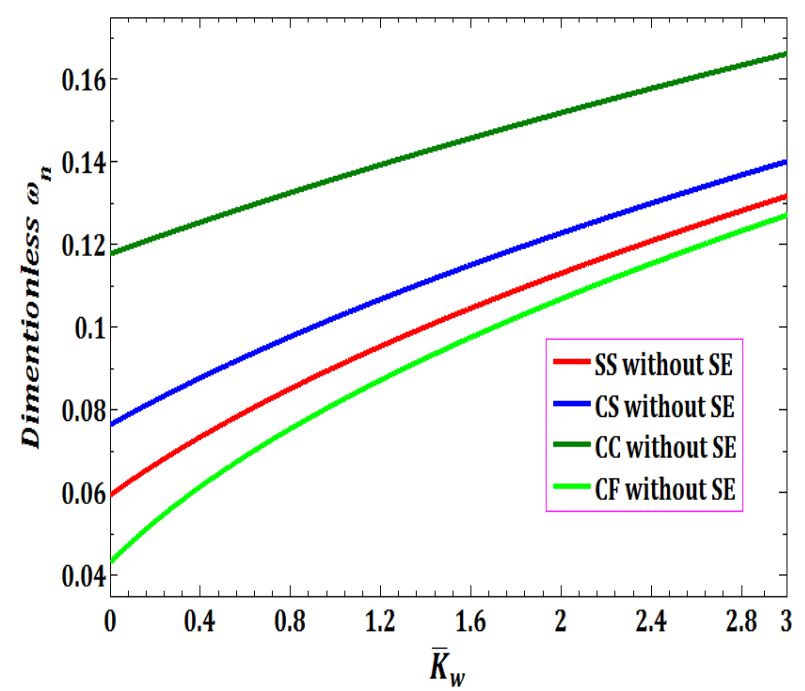

(b)

Fig. 2. The effect of dimensionless stiffness coefficient of Winkler foundation $\bar{K}_{w}$ on dimensionless natural frequencies $\left(\omega_{n}=\bar{\Omega}_{n} R / L\right)$ for (a) with surface effect (b) without surface effect

Furthermore, mode shapes associated to the natural frequencies of cylindrical piezoelectric nano-shell are illustrated in Figure $3(\mathrm{a}-\mathrm{h})$ for different dimensionless stiffness coefficient of Winkler foundation $\bar{K}_{w}$ and for mode number $(m=3, n=$ 4). 


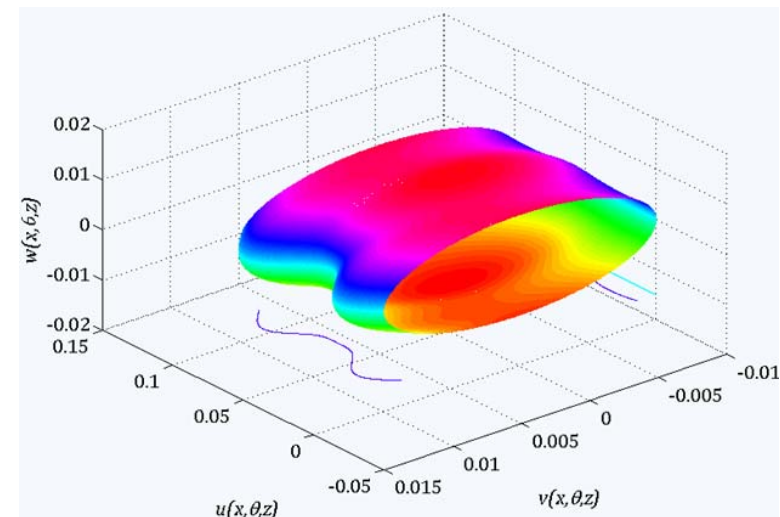

(a) $\mathrm{SS}, \bar{K}_{w}=1$

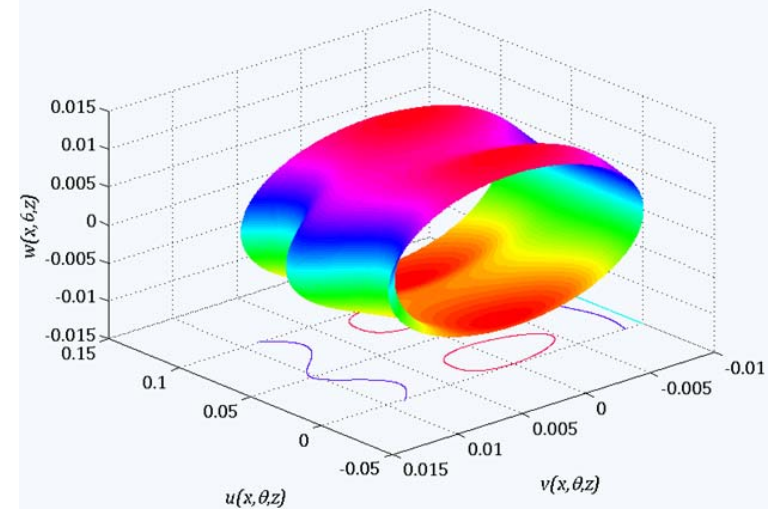

(b) $\mathrm{SS}, \bar{K}_{w}=3$

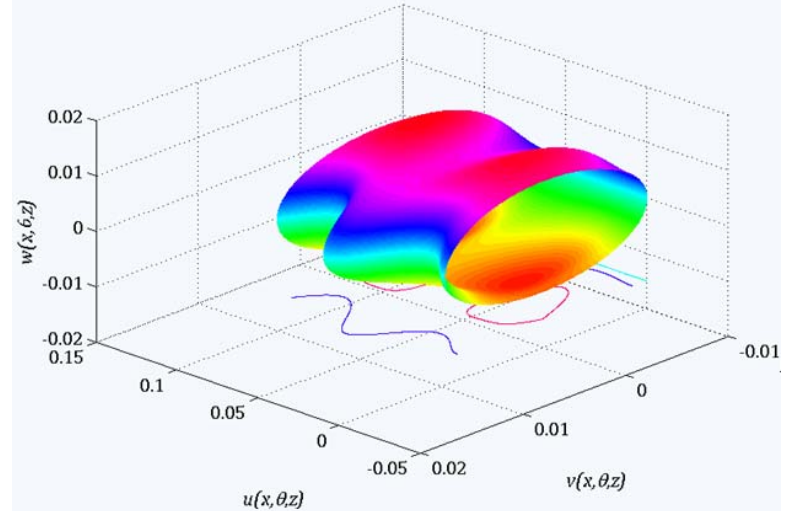

(c) CS, $\bar{K}_{w}=1$

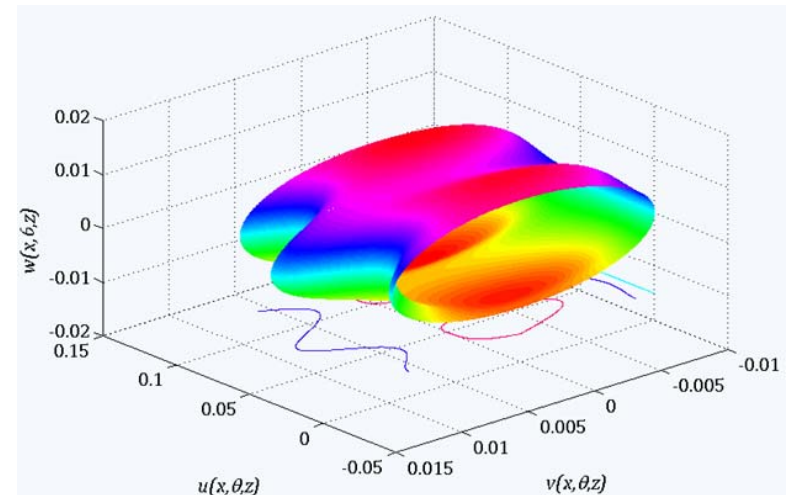

(d) CS, $\bar{K}_{w}=3$

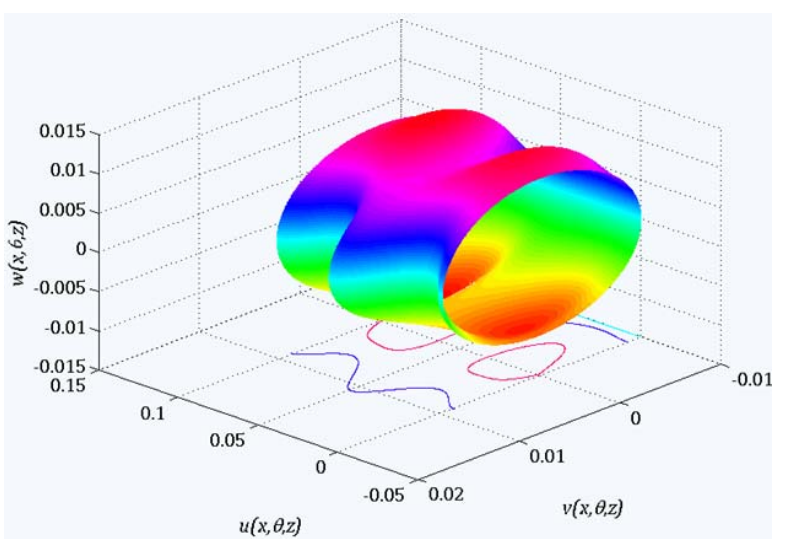

(e) $\mathrm{CC}, \bar{K}_{w}=1$

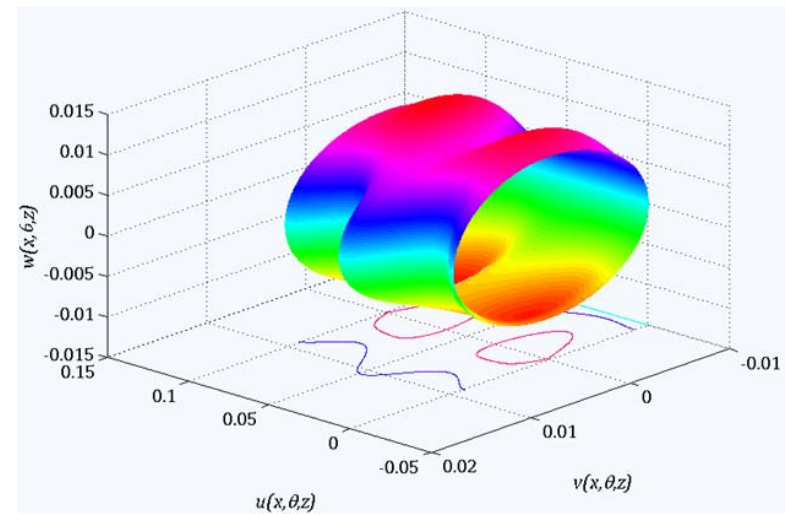

(f) $\mathrm{CC}, \bar{K}_{w}=3$

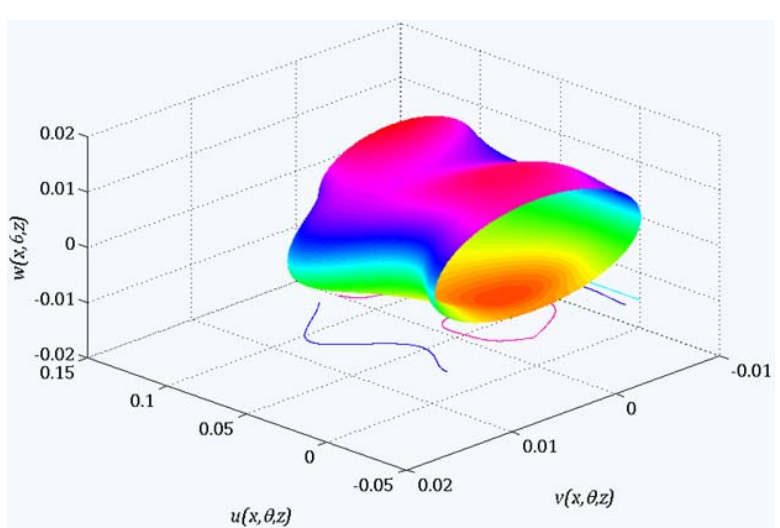

(g) $\mathrm{CF}, \bar{K}_{w}=1$

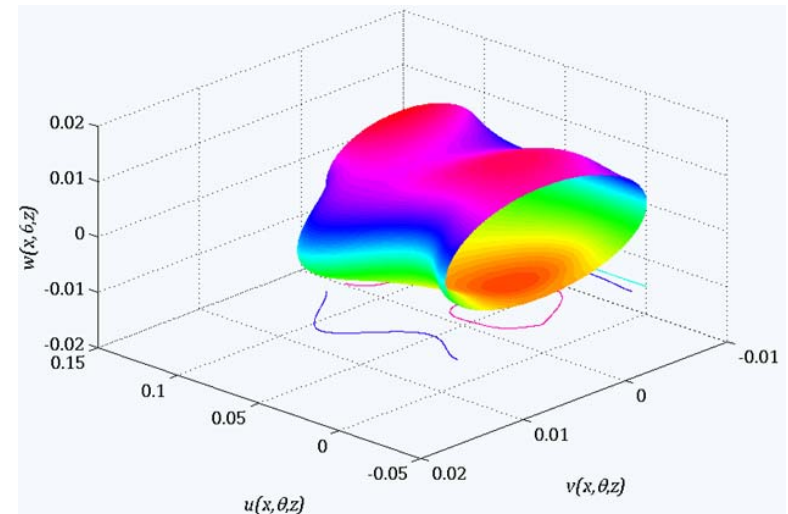

(h) CF, $\bar{K}_{w}=3$ 
Fig. 3. Some selected mode shapes for different dimensionless stiffness coefficient of Winkler foundation $\bar{K}_{w}$

The effect of dimensionless shear layer of Pasternak foundation $\bar{K}_{p}$ on dimensionless natural frequencies of the piezoelectric nano-shell are depicted in Figure 4. It can be seen that for all boundary conditions with and without surface energy effects, with the increase of the nanoshell shear layer coefficient, the fundamental frequency increases.

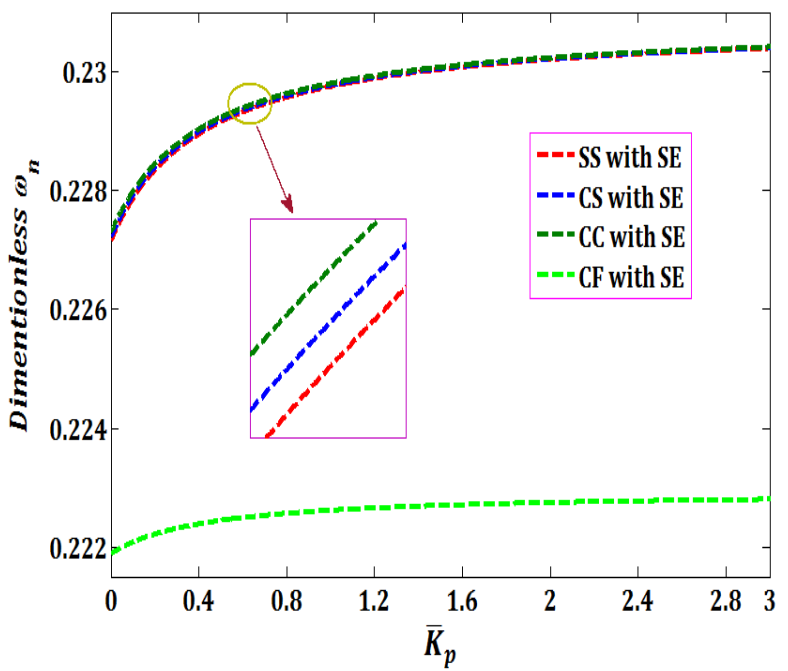

(a)

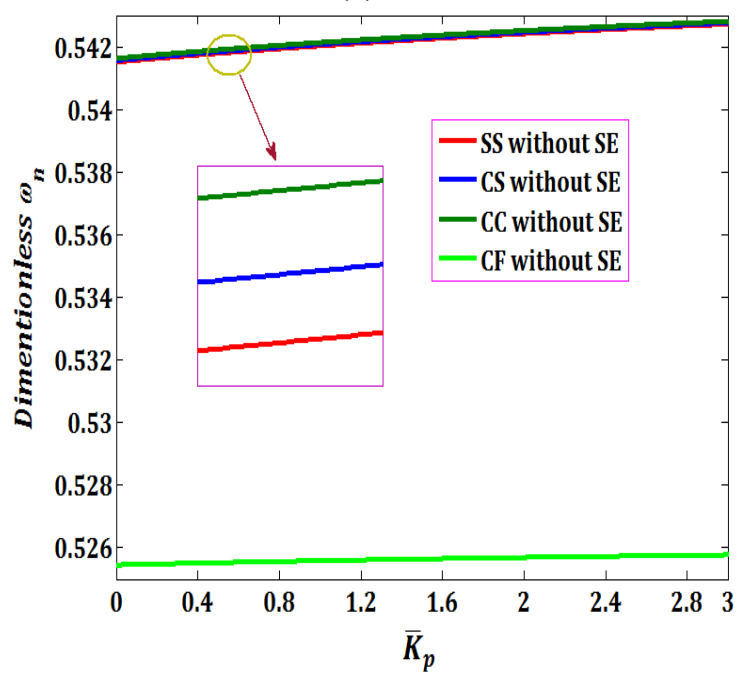

(b)

Fig. 4. The effect of dimensionless shear layer of Pasternak foundation $\bar{K}_{p}$ on dimensionless natural frequencies $\left(\omega_{n}=\bar{\Omega}_{n} R / L\right)$ for (a) with surface effect (b) without surface effect

As can be seen from Figure $4(a, b)$, the natural frequency of the $\mathrm{CC}$ is greater and with boundary conditions SS and CS, there is little difference, and also the natural frequency of the $\mathrm{CF}$ is lower than the rest. It is quite clear that considering the effects of the surface energy will lead to a significant decrease in the natural frequency of the nanoshell. The dimensionless undamped natural frequency of the piezoelectric nanoshell versus length-to-small radius ratio $(L / R)$ is illustrated in Figure $5(\mathrm{a}, \mathrm{b})$.

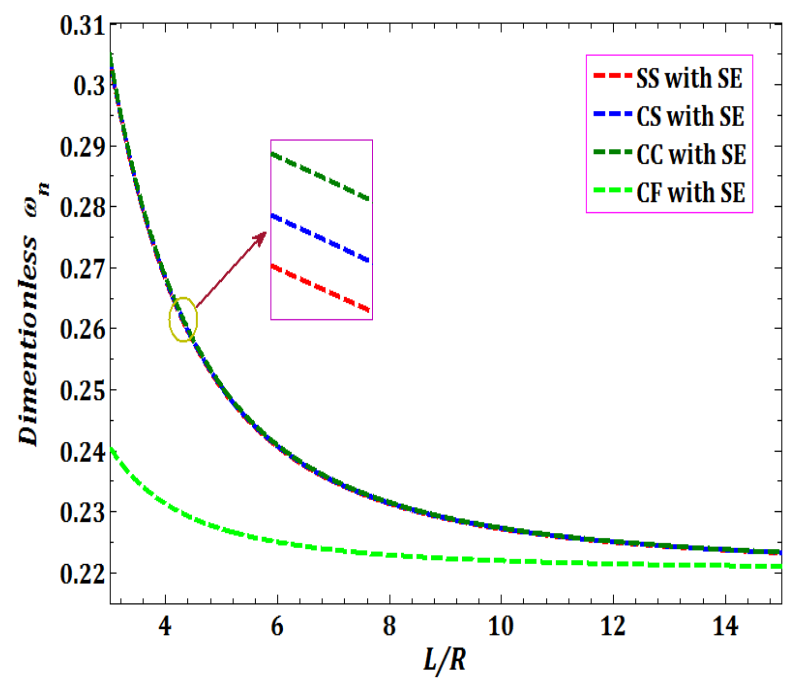

(a)

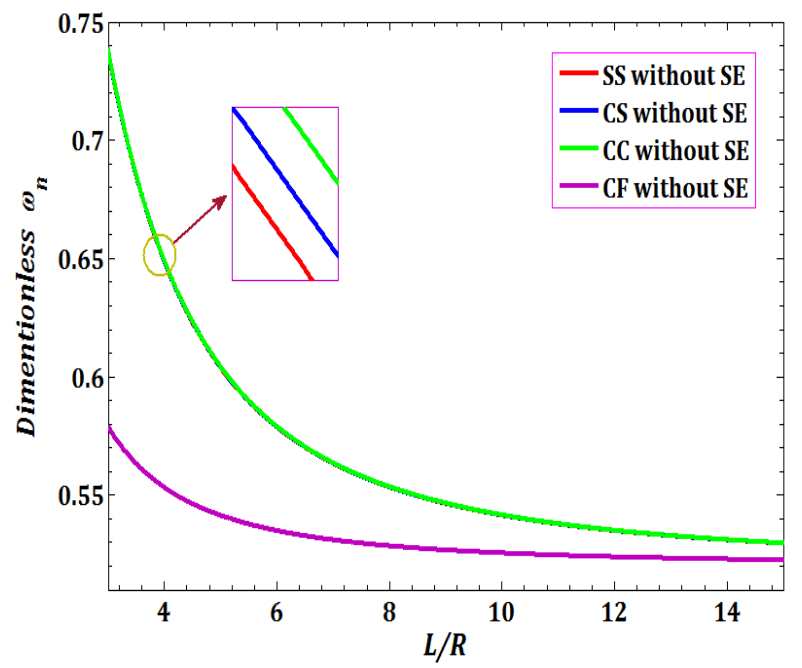

(b)

Fig. 5. The effect of length-to-small radius ratio $L / R$ on dimensionless undamped natural frequencies $\left(\omega_{n}=\bar{\Omega}_{n} R / L\right)$ for (a) with surface effect (b) without surface effect

As it is seen, for all boundary conditions with and without surface energy effects, with the increase of the length-to-small radius ratio $L / R$, the fundamental frequency decreases. In addition, the length-to-small radius ratio of cylindrical shell has 
an important effect on natural frequency. The reason is that a higher $L / R$ ratio lead to a decrease in the nanoshell stiffness, and cause to lower natural frequencies of nanoshells and the vibrational behavior of the shell with the larger $L / R$ ratio is less sensitive to variations of boundary conditions. As can be seen from Figure $5(a, b)$, the natural frequency of the $\mathrm{CC}$ is greater and with boundary conditions SS and CS, there is little difference, and also the natural frequency of the CF is lower than the rest. It is quite clear that considering the effects of the surface energy will lead to a significant decrease in the natural frequency of the nanoshell.

Figure $6(a, b)$ illustrates the effect of thickness shell to small radius ratio $h_{N} / R$ on dimensionless undamped natural frequencies $\left(\omega_{n}=\bar{\Omega}_{n} R / L\right)$ of the piezoelectric nano-shell. It can be seen that for all boundary conditions with and without surface energy effects, with the increase of the nanoshell stiffness coefficient the fundamental frequency increases.

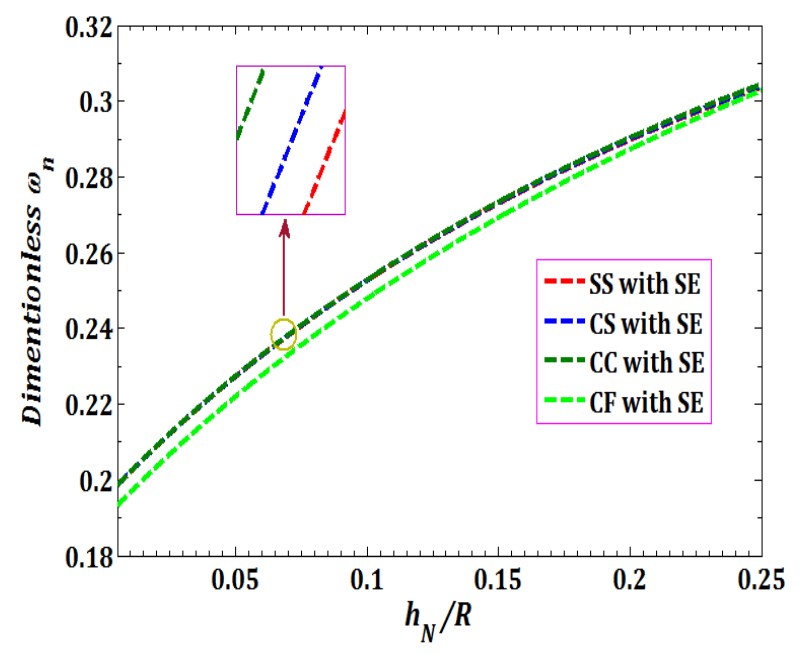

(a)

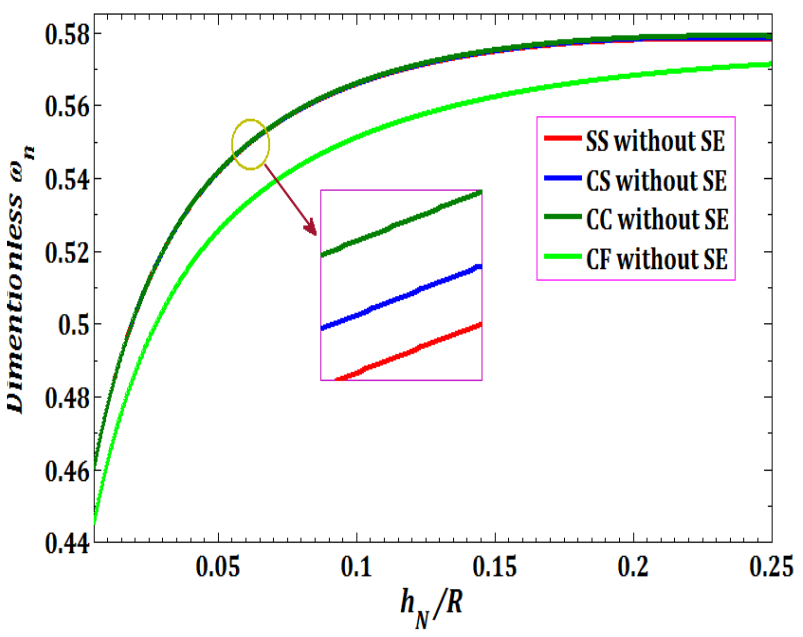

(b)

Fig. 6. The effect of different $h_{N} / R$ ratio on dimensionless undamped natural frequencies $\left(\omega_{n}=\bar{\Omega}_{n} R / L\right)$ for (a) with surface effect (b) without surface effect

As can be seen from Figure $2(a, b)$, the natural frequency of the $\mathrm{CC}$ is greater and with boundary conditions SS and CS, there is little difference, and also the natural frequency of the $\mathrm{CF}$ is lower than the rest. Similar to other previous figures, it is quite clear that considering the effects of the surface energy will lead to a significant decrease in the natural frequency of the nanoshell.

Also the dimensionless undamped natural frequencies $\left(\omega_{n}=\bar{\Omega}_{n} R / L\right)$ of the piezoelectric nano-shell versus thickness piezo to small radius ratio $\left(h_{p} / R\right)$ with and without surface effects are presented in Figure $7(a, b)$. As shown in Figure 7, for all boundary conditions the behavior of the cases with and without surface effects is almost the opposite. It can be shown that for all boundary conditions without surface effects, the fundamental frequency decrease with the increase of the $h_{p} / R$ ratio and vice versa, with surface effects, the fundamental frequency increases with the increase of the $h_{p} / R$ ratio. The reason is that in case of with surface effects a higher $h_{p} / R$ ratio leads to an increase in the nanoshell stiffness, and cause to higher natural frequencies of nanoshells. Similar to other previous results, the natural frequency of the $\mathrm{CC}$ is greater and with boundary conditions SS and $\mathrm{CS}$, there is little difference, and also the natural frequency of the $\mathrm{CF}$ is lower than the rest and also 
the considering the effects of the surface energy will lead to a significant decrease in the natural frequency of the nanoshell.

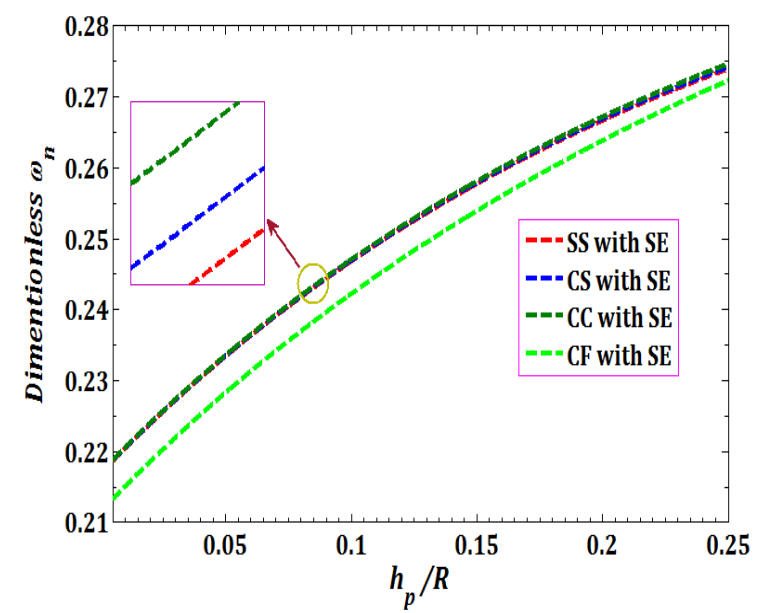

(a)

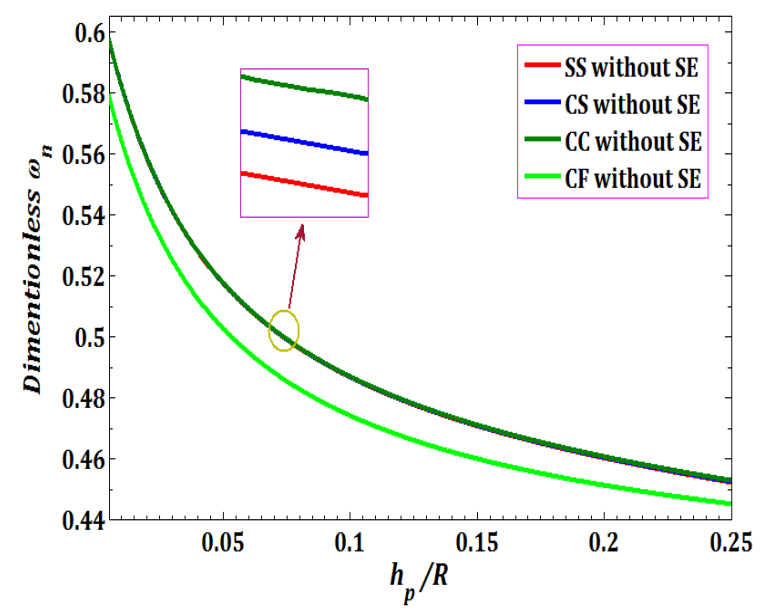

(b)

Fig. 7. The effect of $h_{p} / R$ ratio on dimensionless undamped natural frequencies $\left(\omega_{n}=\bar{\Omega}_{n} R / L\right)$ for (a) with surface effect (b) without surface effect

Figure 8 discusses the effect of the length-to-small radius ratio $(L / R)$ on the dimensionless undamped natural frequency $\omega_{n}$ of simple supported (SS) piezoelectric nanoshells for different values of nonlocal parameter $\mu$ using Eringen nonlocal theory.

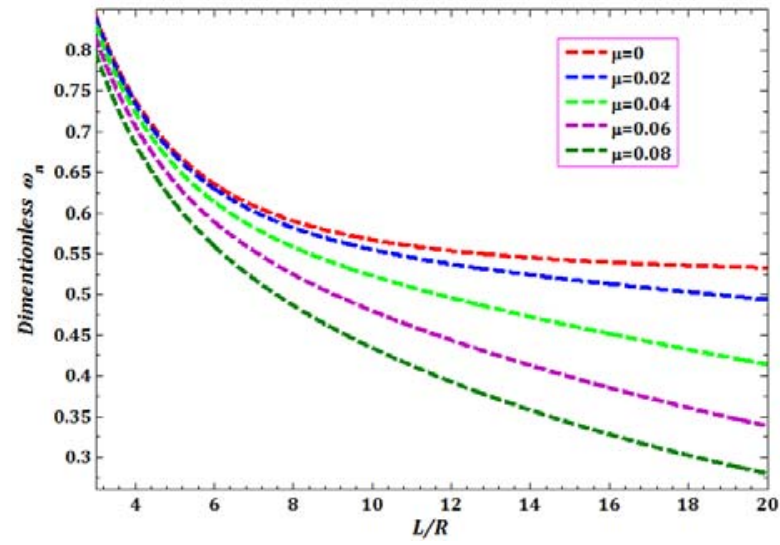

Fig. 8. The effect of length-to-small radius ratio $L / R$ on dimensionless undamped natural frequencies $\left(\omega_{n}=\bar{\Omega}_{n} R / L\right)$ with different values of nonlocal parameter $\mu$ and SS boundary condition

For simple supported (SS) piezoelectric nanoshells, the dimensionless natural frequency decreases with the increase of the ratio $(L / R)$. As shown in Figure 8 , the natural frequency decreases with increase of nonlocal parameter $\mu$. The reason is that a higher nonlocal parameter $\mu$ leads to a decrease in the nanoshell stiffness, and cause to lower natural frequencies of nanoshell.

And finally, the effect of the small radius ratio $h_{N} / R$ on dimensionless natural frequencies $\left(\omega_{n}=\bar{\Omega}_{n} R / L\right)$ of the simple supported (SS) piezoelectric nanoshell for different values of nonlocal parameter $\mu$ are shown in Figure 9 using Eringen nonlocal theory. As shown in Figure 9, the dimensionless natural frequency increase with the increase of the ratio $h_{N} / R$. Also, the natural frequency decreases with increase of nonlocal parameter $\mu$. The reason is that a higher nonlocal parameter $\mu$ leads to a decrease in the nanoshell stiffness, and cause to lower natural frequencies of nanoshell. 


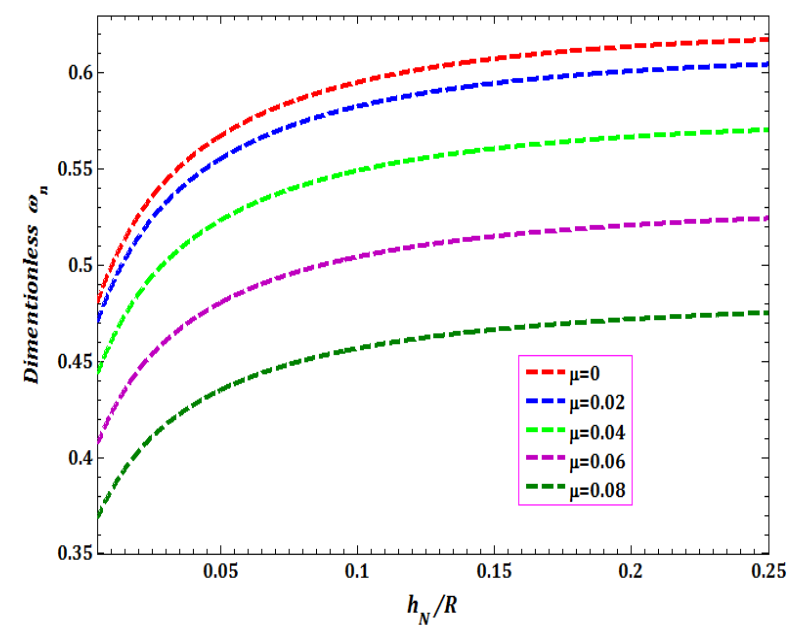

Fig. 9. Dimensionless undamped natural frequency $\left(\omega_{n}=\bar{\Omega}_{n} R / L\right)$ of the piezoelectric nano-shell versus $\left(h_{N} / R\right)$ ratio for different value of the dimensionless nonlocal parameter $\mu$

\section{Conclusion}

Vibration analysis of piezoelectric cylindrical nanoshell subjected to visco-Pasternak medium with arbitrary boundary conditions is investigated by accounting for the simultaneous effects of the surface elasticity and the different material scale parameter. To this end, Eringen nonlocal theory and Gurtin-Murdoch surface/interface theory and Donnell's theory are used. The governing equations and boundary conditions are derived using Hamilton's principle and also, assumed mode method combined with Euler-Lagrange method is used for discretizing equations of motion. The viscoelastic nanoshell medium is modeled as viscoPasternak. A variety of new vibration results including natural frequencies with and without nonlocal and surface energy effects for piezoelectric cylindrical nano-shell with nonclassical restraints as well as different material parameters are presented, which may serve as benchmark solution for future researches. The convergence, accuracy and reliability of the current formulation are validated by comparisons with existing experimental and numerical results published in the literature, with excellent agreements achieved. Also, the effects of nonlocality, surface energy, nanoshell radius, circumferential wavenumber, nanoshell damping coefficient, and foundation damping are accurately studied on frequencies and mode shapes of piezoelectric cylindrical nanoshell.

Some conclusions are obtained from this study:

With comparing the previously published in the literature, the present results agree very well with the reference solutions, which indicates that the methods are suitable and of high accuracy for free vibration analysis of cylindrical nanoshell.

$\checkmark$ For all boundary conditions for as the number of polynomial terms, $N$, is increased, the value of the frequency parameter, $\omega_{n}$, converges rapidly and also the convergence mode number for both cases of the paper results, i.e., GurtinMurdoch surface/interface and Eringen nonlocal theories is $n=2$ and $m=2$.

$\checkmark$ With the increase of the dimensionless stiffness coefficient of Winkler foundation $\bar{K}_{w}$, for all boundary conditions with and without surface energy effects, the fundamental frequency increases.

$\checkmark$ For all boundary conditions with and without surface energy effects, with the increase of the dimensionless shear layer of Pasternak foundation $\bar{K}_{p}$, the fundamental frequency increases.

$\checkmark$ With the increase of the $L / R$ ratio, the fundamental frequency for all boundary conditions with and without surface effects decreases.

$\checkmark$ For all boundary conditions with and without surface energy effects, with the increase of the nanoshell stiffness coefficient $h_{N} / R$ the fundamental frequency increases.

$\checkmark$ For all boundary conditions, with considering of ratio $\left(h_{p} / R\right)$, the behavior of the cases with and without surface effects is almost the opposite. In case of without surface affects, the fundamental frequency decrease with the increase of the $h_{p} / R$ ratio and vice versa, with surface effects, the fundamental frequency increases with the increase of the $h_{p} / R$ ratio.

$\checkmark$ In all mention results, the natural frequency of the CC is greater and with boundary conditions SS and CS, there is little difference, and also the natural frequency of the CF is lower than the rest. 
For all boundary conditions, the dimensionless natural frequency decreases with the increase of the ratio $(L / R)$ and the nonlocal parameter $\mu$.

$\checkmark$ For all boundary conditions, the dimensionless natural frequency increase with the increase of the ratio $h_{N} / R$ and also because of a higher nonlocal parameter $\mu$ leads to a decrease in the nanoshell stiffness, the natural frequency decreases with increase of nonlocal parameter $\mu$.

\section{Conflict of interest}

The authors report no conflict of interest.

\section{Funding Acknowledgement}

'This research received no specific grant from any funding agency in the public, commercial, or notfor-profit sectors'.

\section{Appendix 1}

$$
\begin{aligned}
& \alpha_{1}=\frac{1}{m_{3}} \bar{A}_{11}, \alpha_{2}=\frac{m_{0}}{m_{3}}\left(\bar{A}_{12}+\bar{A}_{21}\right) \text {, } \\
& \alpha_{3}=\frac{m_{0}}{m_{3}}\left(\bar{A}_{12}+\bar{A}_{21}\right), \alpha_{4}=\frac{m_{0}^{2}}{m_{3}} \bar{A}_{22} \text {, } \\
& \alpha_{5}=\frac{m_{0}^{2}}{m_{3}}\left(\bar{A}_{22}-\bar{\tau}_{0}^{s_{1}}-\bar{\tau}_{0}^{s_{2}}\right), \\
& \alpha_{6}=\frac{m_{0}^{2}}{m_{3}}\left(2 \bar{A}_{22}-\bar{\tau}_{0}^{s_{1}}-\bar{\tau}_{0}^{s_{2}}\right), \alpha_{7}=\frac{m_{0}^{2}}{m_{3}} \bar{A}_{66} \text {, } \\
& \alpha_{8}=\frac{1}{m_{3}} \bar{A}_{66}, \alpha_{9}=\frac{2 m_{0}}{m_{3}} \bar{A}_{66} \text {, } \\
& \alpha_{10}=\frac{1}{m_{1} m_{3}}\left(\bar{F}_{11}-2 \bar{B}_{11}\right) \text {, } \\
& \alpha_{11}=\frac{m_{0} m_{2}}{m_{3}}\left(\bar{F}_{11}-\bar{B}_{12}-\bar{B}_{21}\right) \text {, } \\
& \alpha_{12}=\frac{m_{2}}{m_{3}}\left(\bar{F}_{11}-\bar{B}_{12}-\bar{B}_{21}\right) \\
& , \alpha_{13}=\frac{m_{2}}{m_{3}}\left(\bar{F}_{11}-\bar{B}_{12}-\bar{B}_{21}\right) \text {, } \\
& \alpha_{14}=\frac{m_{0}^{2} m_{2}}{m_{3}}\left(\bar{F}_{11}-2 \bar{B}_{22}\right) \text {, } \\
& \alpha_{15}=\frac{m_{0}^{2} m_{2}}{m_{3}}\left(\bar{F}_{11}-2 \bar{B}_{22}+\bar{\tau}_{0}^{s_{2}}\left(1+m_{4}\right)-\bar{\tau}_{0}^{s_{1}}\right) \text {, } \\
& \alpha_{16}=-\frac{4 m_{0} m_{2}}{m_{3}} \bar{B}_{66}, \alpha_{17}=-\frac{4 m_{2}}{m_{3}} \bar{B}_{66} \text {, }
\end{aligned}
$$

$$
\begin{aligned}
\alpha_{18}= & \frac{1}{m_{1}^{2} m_{3}}\left(\bar{D}_{11}-\bar{E}_{11}\right), \\
\alpha_{19}= & \frac{m_{2}^{2}}{m_{3}}\left(\bar{D}_{12}+\bar{D}_{21}-2 \bar{E}_{11}\right), \\
\alpha_{20}= & \frac{m_{0}^{2} m_{2}^{2}}{m_{3}}\left(\bar{D}_{22}-\bar{E}_{11}\right), \alpha_{21}=\frac{4 m_{2}^{2}}{m_{3}} \bar{D}_{66}, \\
\alpha_{22}= & \frac{m_{0} m_{1}}{m_{3}}\left(\bar{\tau}_{0}^{s_{1}}+\bar{\tau}_{0}^{s_{2}}-\bar{N}_{\theta p}\right), \\
\alpha_{23}= & \frac{m_{1}}{m_{3}}\left(\bar{\tau}_{0}^{s_{1}}+\bar{\tau}_{0}^{s_{2}}-\bar{N}_{x p}\right), \\
\alpha_{24}= & \frac{m_{0} m_{1}}{m_{3}}\left(\bar{\tau}_{0}^{s_{1}}+\bar{\tau}_{0}^{s_{2}}-\bar{N}_{\theta p}\right), \\
\alpha_{25}= & \frac{1}{m_{3}}\left(\bar{M}_{x p}+\bar{\tau}_{0}^{s_{1}}-\bar{\tau}_{0}^{s_{2}}\left(1+m_{4}\right)\right), \\
\alpha_{26}= & \frac{m_{0}^{2}}{m_{3}}\left(\bar{M}_{\theta p}+\bar{\tau}_{0}^{s_{1}}-\bar{\tau}_{0}^{s_{2}}\left(1+m_{4}\right)\right), \\
\alpha_{27}= & -\frac{1}{2 m_{1}^{2} m_{3}} \bar{G}_{11}^{*}, \alpha_{28}=-\frac{m_{2}^{2}}{2 m_{3}} \bar{G}_{11}^{*}, \\
\alpha_{29}= & \frac{1}{2 m_{1} m_{3}} \bar{J}_{11}^{*}, \alpha_{30}=\frac{m_{2}}{2 m_{3}} \bar{J}_{11}^{*}, \alpha_{31}=\frac{m_{2}}{2 m_{3}} \bar{J}_{11}^{*},
\end{aligned}
$$

\section{Appendix 2}

$$
\begin{aligned}
& \beta_{1}=\frac{1}{m_{3}} \bar{A}_{11}, \beta_{2}=\frac{m_{0}^{2}}{m_{3}} \bar{A}_{66} \\
& \beta_{3}=\frac{m_{0}}{m_{3}}\left(\bar{A}_{12}+\bar{A}_{66}\right), \beta_{4}=\frac{m_{0}}{m_{3}} \bar{A}_{12} \\
& \beta_{5}=-\frac{1}{m_{1} m_{3}} \bar{B}_{11}, \beta_{6}=-\frac{m_{0} m_{2}}{m_{3}}\left(\bar{B}_{12}+2 \bar{B}_{66}\right), \\
& \beta_{7}=\frac{m_{0}^{2}}{m_{3}} \bar{A}_{66}, \beta_{8}=\frac{m_{0}}{m_{3}} \bar{A}_{21}, \beta_{9}=\frac{1}{m_{3}} \bar{A}_{66} \\
& \beta_{10}=\frac{m_{0}^{2}}{m_{3}} \bar{A}_{22}, \beta_{11}=\frac{m_{0}^{2}}{m_{3}} \bar{A}_{22} \\
& \beta_{12}=-\frac{m_{2}}{m_{3}}\left(\bar{B}_{21}+2 \bar{B}_{66}\right), \beta_{13}=-\frac{m_{0}^{2} m_{2}}{m_{3}} \bar{B}_{22} \\
& \beta_{14}=-\frac{m_{0}}{m_{3}} \bar{A}_{21}, \beta_{15}=\frac{1}{m_{1} m_{3}} \bar{B}_{11}, \\
& \beta_{16}=\frac{m_{0} m_{2}}{m_{3}}\left(\bar{B}_{21}+2 \bar{B}_{66}\right), \beta_{17}=-\frac{m_{0}^{2}}{m_{3}} \bar{A}_{22}, \\
& \beta_{18}=\frac{m_{2}}{m_{3}}\left(\bar{B}_{21}+2 \bar{B}_{66}\right), \beta_{19}=\frac{m_{0}^{2} m_{2}}{m_{3}} \bar{B}_{22} \\
& \beta_{20}=-\frac{m_{0}^{2}}{m_{3}} \bar{A}_{22}, \beta_{21}=\frac{m_{2}}{m_{3}}\left(\bar{B}_{12}+\bar{B}_{21}\right) \\
& \beta_{22}=\frac{2 m_{0}^{2} m_{2}}{m_{3}} \bar{B}_{22}, \beta_{23}=\frac{1}{m_{1}^{2} m_{3}} \bar{D}_{11} \\
& \beta_{24}=-\frac{m_{2}^{2}}{m_{3}}\left(\bar{D}_{12}+\bar{D}_{21}+4 \bar{D}_{66}\right)
\end{aligned}
$$




$$
\beta_{25}=-\frac{m_{0}^{2} m_{2}^{2}}{m_{3}} \bar{D}_{22}, \beta_{26}=\frac{m_{0} m_{1}}{m_{3}} \bar{N}_{\theta p},
$$

\section{Appendix 3}

$\bar{\beta}_{1}=\frac{1}{m_{3}} \bar{A}_{11}, \bar{\beta}_{2}=\frac{m_{0}}{m_{3}} \bar{A}_{12}, \overline{\beta_{3}}=\frac{m_{0}}{m_{3}} \bar{A}_{12}$,

$\bar{\beta}_{4}=-\frac{1}{m_{1} m_{3}} \bar{B}_{11}, \bar{\beta}_{5}=-\frac{m_{0} m_{2}}{m_{3}} \bar{B}_{12}$,

$\bar{\beta}_{6}=-\frac{1}{m_{1} m_{3}} \bar{N}_{x p}, \bar{\beta}_{7}=\frac{m_{0}}{m_{3}} \bar{A}_{66}, \bar{\beta}_{8}=\frac{1}{m_{3}} \bar{A}_{66}$,

$\bar{\beta}_{9}=-\frac{2 m_{2}}{m_{3}} \bar{B}_{66}, \bar{\beta}_{10}=\frac{m_{0}}{m_{3}} \bar{A}_{66}$,

$\bar{\beta}_{11}=\frac{1}{m_{3}} \bar{A}_{66}, \bar{\beta}_{12}=-\frac{2 m_{2}}{m_{3}} \bar{B}_{66}, \bar{\beta}_{13}=\frac{1}{m_{3}} \bar{A}_{21}$,

$\bar{\beta}_{14}=\frac{m_{0}}{m_{3}} \bar{A}_{22}, \bar{\beta}_{15}=\frac{m_{0}}{m_{3}} \bar{A}_{22}, \bar{\beta}_{16}=-\frac{1}{m_{1} m_{3}} \bar{B}_{21}$,

$\bar{\beta}_{17}=-\frac{m_{0} m_{2}}{m_{3}} \bar{B}_{22}, \bar{\beta}_{18}=-\frac{m_{1}}{m_{3}} \bar{N}_{\theta p}$,

$\bar{\beta}_{19}=\frac{1}{m_{1} m_{3}} \bar{B}_{11}, \bar{\beta}_{20}=\frac{m_{0} m_{2}}{m_{3}} \bar{B}_{66}$,

$\bar{\beta}_{21}=\frac{m_{2}}{m_{3}}\left(\bar{B}_{12}+\bar{B}_{66}\right), \bar{\beta}_{22}=\frac{m_{2}}{m_{3}} \bar{B}_{12}$,

$\bar{\beta}_{23}=-\frac{1}{m_{1}^{2} m_{3}} \bar{D}_{11}, \bar{\beta}_{24}=-\frac{m_{2}^{2}}{m_{3}}\left(\bar{D}_{12}+2 \bar{D}_{66}\right)$,

$\bar{\beta}_{25}=\frac{m_{2}}{m_{3}}\left(\bar{B}_{21}+\bar{B}_{66}\right), \bar{\beta}_{26}=\frac{1}{m_{1} m_{3}} \bar{B}_{66}$,

$\bar{\beta}_{27}=\frac{m_{0} m_{2}}{m_{3}} \bar{B}_{22}, \bar{\beta}_{28}=\frac{m_{0} m_{2}}{m_{3}} \bar{B}_{22}$,

$\bar{\beta}_{29}=-\frac{m_{0} m_{2}^{2}}{m_{3}} \bar{D}_{22}$,

$\bar{\beta}_{30}=-\frac{m_{2}}{m_{1} m_{3}}\left(\bar{D}_{21}+2 \bar{D}_{66}\right)$,

$\bar{\beta}_{31}=\frac{1}{m_{1} m_{3}} \bar{B}_{11}, \bar{\beta}_{32}=\frac{m_{2}}{m_{3}} \bar{B}_{12}$,

$\bar{\beta}_{33}=\frac{m_{2}}{m_{3}} \bar{B}_{12}, \bar{\beta}_{34}=-\frac{1}{m_{1}^{2} m_{3}} \bar{D}_{11}$,

$\bar{\beta}_{35}=-\frac{m_{2}^{2}}{m_{3}} \bar{D}_{12}, \bar{\beta}_{36}=-\frac{1}{m_{3}} \bar{M}_{x p}$

$\bar{\beta}_{37}=\frac{m_{2}}{m_{3}} \bar{B}_{66}, \bar{\beta}_{38}=\frac{1}{m_{1} m_{3}} \bar{B}_{12}$,

$\bar{\beta}_{39}=-\frac{2 m_{2}}{m_{1} m_{3}} \bar{D}_{66}, \bar{\beta}_{40}=\frac{m_{0} m_{2}}{m_{3}} \bar{B}_{66}$,

$\bar{\beta}_{41}=\frac{m_{2}}{m_{3}} \bar{B}_{66}, \bar{\beta}_{42}=\frac{2 m_{2}^{2}}{m_{3}} \bar{D}_{66}, \bar{\beta}_{43}=\frac{m_{2}}{m_{3}} \bar{B}_{21}$,

$\bar{\beta}_{44}=\frac{m_{0} m_{2}}{m_{3}} \bar{B}_{22}, \bar{\beta}_{45}=\frac{m_{0} m_{2}}{m_{3}} \bar{B}_{22}$,

$\bar{\beta}_{46}=-\frac{m_{2}}{m_{1} m_{3}} \bar{D}_{21}, \bar{\beta}_{47}=-\frac{m_{0} m_{2}^{2}}{m_{3}} \bar{D}_{22}$ $\bar{\beta}_{48}=-\frac{m_{0}}{m_{3}} \bar{M}_{x p}$

\section{Appendix 4}

$(M)_{u L}^{u}=\iint \chi_{i} \chi_{e} \vartheta_{j} \vartheta_{f} d \xi d \theta$,

$(M)_{u L}^{w}=\frac{1}{2} \alpha_{29} \iint \chi_{\mathrm{e}}^{\prime} \beta_{o} \vartheta_{f} \psi_{l} d \xi d \theta$

$(K)_{u L}^{u}=\iint\left(\alpha_{1} \chi_{\mathrm{i}}^{\prime} \chi_{\mathrm{e}}^{\prime} \vartheta_{j} \vartheta_{f}+\alpha_{7} \chi_{i} \chi_{e} \vartheta_{j}^{\prime} \vartheta_{f}^{\prime}\right) d \xi d \theta$

$(K)_{u L}^{v}=\frac{1}{2} \iint\left(\alpha_{2} \chi_{i}^{\prime} \phi_{k} \vartheta_{j} \alpha_{l}^{\prime}+\alpha_{9} \chi_{i} \phi_{k}^{\prime} \vartheta_{j}^{\prime} \alpha_{l}\right) d \xi d \theta$

$(K)_{u L}^{w}$

$=\frac{1}{2} \iint\left(\begin{array}{c}\alpha_{3} \chi_{\mathrm{i}}^{\prime} \beta_{o} \vartheta_{j} \psi_{p}+\alpha_{10} \chi_{\mathrm{i}}^{\prime} \beta_{0}^{\prime \prime} \vartheta_{j} \psi_{p} \\ +\alpha_{11} \chi_{\mathrm{i}}^{\prime} \beta_{o} \vartheta_{j} \psi_{p}^{\prime \prime}+\alpha_{16} \chi_{i} \beta_{0}^{\prime} \vartheta_{j}^{\prime} \psi_{p}^{\prime}\end{array}\right) d \xi d \theta$

$\bar{F}_{u p L}=\frac{1}{2} \alpha_{23} \iint \chi_{\mathrm{e}}^{\prime} \vartheta_{i} d \xi d \theta,(M)_{v L}^{v}$

$=\iint \phi_{k} \phi_{q} \alpha_{l} \alpha_{g} d \xi d \theta$

$(M)_{v L}^{w}=\frac{1}{2} \alpha_{30} \iint \phi_{q} \beta_{o} \alpha_{f}^{\prime} \psi_{l} d \xi d \theta,(K)_{v L}^{u}$

$=\frac{1}{2} \iint\left(\alpha_{2} \phi_{k} \chi_{\mathrm{i}}^{\prime} \alpha_{l}^{\prime} \vartheta_{j}+\alpha_{9} \phi_{k}^{\prime} \chi_{i} \alpha_{l} \vartheta_{j}^{\prime}\right) d \xi d \theta$

$(K)_{v L}^{v}=\iint\left(\alpha_{4} \phi_{k} \phi_{q} \alpha_{l}^{\prime} \alpha_{g}^{\prime}+\alpha_{8} \phi_{k}^{\prime} \phi_{q}^{\prime} \alpha_{l} \alpha_{g}\right) d \xi d \theta$

$(K)_{v L}^{w}=$

$\frac{1}{2} \iint\left(\begin{array}{c}\alpha_{6} \phi_{k} \beta_{o} \alpha_{l}^{\prime} \psi_{p}+\alpha_{12} \phi_{k} \beta_{0}^{\prime \prime} \alpha_{l}^{\prime} \psi_{p} \\ +\alpha_{14} \phi_{k} \beta_{o} \alpha_{l}^{\prime} \psi_{p}^{\prime \prime}+\alpha_{17} \phi_{k}^{\prime} \beta_{0}^{\prime} \alpha_{l} \psi_{p}^{\prime}\end{array}\right) d \xi d \theta$

$\bar{F}_{v p L}=\frac{1}{2} \alpha_{24} \iint \phi_{q} \alpha_{f}^{\prime} d \xi d \theta$

$(M)_{w L}^{w}=$

$\frac{1}{2} \iint\left(\begin{array}{c}2 \beta_{r} \beta_{o} \psi_{s} \psi_{p}+\alpha_{27} \beta_{r}^{\prime \prime} \beta_{o} \psi_{s} \psi_{p} \\ +\alpha_{28} \beta_{r} \beta_{o} \psi_{s}^{\prime \prime} \psi_{p}+\alpha_{31} \beta_{r} \beta_{o} \psi_{s} \psi_{p}\end{array}\right) d \xi d \theta$

$(C)_{w L}^{w}=\bar{C}_{w} \iint \beta_{r} \beta_{o} \psi_{s} \psi_{p} d \xi d \theta$

$(K)_{w L}^{u}=$

$\frac{1}{2} \iint\left(\begin{array}{c}\alpha_{3} \beta_{o} \chi_{\mathrm{i}}^{\prime} \psi_{p} \vartheta_{j}+\alpha_{10} \beta_{0}^{\prime \prime} \chi_{\mathrm{i}}^{\prime} \psi_{p} \vartheta_{j} \\ +\alpha_{11} \beta_{o} \chi_{\mathrm{i}}^{\prime} \psi_{p}^{\prime \prime} \vartheta_{j}+\alpha_{16} \beta_{0}^{\prime} \chi_{i} \psi_{p}^{\prime} \vartheta_{j}^{\prime}\end{array}\right) d \xi d \theta$

$(K)_{w L}^{v}=$

$\frac{1}{2} \iint\left(\begin{array}{c}\alpha_{6} \beta_{o} \phi_{k} \psi_{p} \alpha_{l}^{\prime}+\alpha_{12} \beta_{0}^{\prime \prime} \phi_{k} \psi_{p} \alpha_{l}^{\prime} \\ +\alpha_{14} \beta_{o} \phi_{k} \psi_{p}^{\prime \prime} \alpha_{l}^{\prime}+\alpha_{17} \beta_{0}^{\prime} \phi_{k}^{\prime} \psi_{p}^{\prime} \alpha_{l}\end{array}\right) d \xi d \theta$

$(K)_{w L}^{w}=$ 
$\frac{1}{2} \iint\left(\begin{array}{c}2 \alpha_{5} \beta_{o} \beta_{r} \psi_{s} \psi_{p}+\alpha_{13} \beta_{0}^{\prime \prime} \beta_{r} \psi_{s} \psi_{p} \\ +\alpha_{13} \beta_{o} \beta_{r}^{\prime \prime} \psi_{s} \psi_{p}+\alpha_{15} \beta_{o} \beta_{r} \psi_{s} \psi_{p}^{\prime \prime} \\ +\alpha_{15} \beta_{o} \beta_{r} \psi_{s}^{\prime \prime} \psi_{p}+2 \alpha_{18} \beta_{0}^{\prime \prime} \beta_{r}^{\prime \prime} \psi_{s} \psi_{p} \\ +\alpha_{19} \beta_{0}^{\prime \prime} \beta_{r} \psi_{s} \psi_{p}^{\prime \prime}+\alpha_{19} \beta_{o} \beta_{r}^{\prime \prime} \psi_{s}^{\prime \prime} \psi_{p} \\ +2 \alpha_{20} \beta_{o} \beta_{r} \psi_{s}^{\prime \prime} \psi_{p}^{\prime \prime}+2 \alpha_{21} \beta_{0}^{\prime} \beta_{r}^{\prime} \psi_{s}^{\prime} \psi_{p}^{\prime} \\ +2 \bar{k}_{w} \beta_{o} \beta_{r} \psi_{s} \psi_{p}-2 \bar{k}_{p} \beta_{0}^{\prime \prime} \beta_{r} \psi_{s} \psi_{p} \\ -2 \bar{k}_{p} m_{0}^{2} \beta_{o} \beta_{r} \psi_{s}^{\prime \prime} \psi_{p}\end{array}\right) d \xi d \theta$

$\bar{F}_{w p L}=$

$\frac{1}{2} \iint\left(\alpha_{22} \beta_{r} \psi_{s}+\alpha_{25} \beta_{r}^{\prime \prime} \psi_{s}+\alpha_{26} \beta_{r} \psi_{s}^{\prime \prime}\right) d \xi d \theta$

\section{Appendix 5}

$(M)_{u H}^{u}=\iint\left(\begin{array}{c}\chi_{i} \chi_{e} \vartheta_{j} \vartheta_{f}-\mu^{2} \chi_{i} \chi_{\mathrm{e}}^{\prime \prime} \vartheta_{j} \vartheta_{f} \\ -\mu^{2} m_{0}^{2} \chi_{i} \chi_{e} \vartheta_{j} \vartheta_{f}^{\prime \prime}\end{array}\right) d \xi d \theta$

$(K)_{u H}^{u}=$

$-\iint\left(\beta_{1} \chi_{i} \chi_{\mathrm{e}}^{\prime \prime} \vartheta_{j} \vartheta_{f}+\beta_{2} \chi_{i} \chi_{e} \vartheta_{j} \vartheta_{f}^{\prime \prime}\right) d \xi d \theta$

$\left(K_{b c}\right)_{u H}^{u}=\left.\bar{\beta}_{1}\left(\chi_{i} \chi_{\mathrm{e}}^{\prime}\right)\right|_{0} ^{1} \int \vartheta_{j} \vartheta_{f} d \theta$

$+\left.\bar{\beta}_{7}\left(\vartheta_{j} \vartheta_{f}^{\prime}\right)\right|_{0} ^{2 \pi} \int \chi_{i} \chi_{e} d \xi$

$(K)_{u H}^{v}=-\iint\left(\beta_{3} \chi_{i} \phi_{q}^{\prime} \vartheta_{j} \alpha_{l}^{\prime}\right) d \xi d \theta$

$\left(K_{b c}\right)_{u H}^{v}=$

$\left.\bar{\beta}_{2}\left(\chi_{i} \phi_{q}\right)\right|_{0} ^{1} \int \vartheta_{j} \alpha_{l}^{\prime} d \theta+\left.\bar{\beta}_{8}\left(\vartheta_{j} \alpha_{l}\right)\right|_{0} ^{2 \pi} \int \chi_{i} \phi_{q}^{\prime} d \xi$

$(K)_{u H}^{w}=$

$-\iint\left(\begin{array}{c}\beta_{4} \chi_{i} \beta_{0}^{\prime} \vartheta_{j} \psi_{p}+\beta_{5} \chi_{i} \beta_{0}^{\prime \prime \prime} \vartheta_{j} \psi_{p} \\ +\beta_{6} \chi_{i} \beta_{0}^{\prime} \vartheta_{j} \psi_{p}^{\prime \prime}\end{array}\right) d \xi d \theta$

$\left(K_{b c}\right)_{u H}^{w}=\left.\bar{\beta}_{3}\left(\chi_{i} \beta_{o}\right)\right|_{0} ^{1} \int \vartheta_{j} \psi_{p} d \theta$

$+\left.\bar{\beta}_{4}\left(\chi_{i} \beta_{0}^{\prime \prime}\right)\right|_{0} ^{1} \int \vartheta_{j} \psi_{p} d \theta+\left.\bar{\beta}_{5}\left(\chi_{i} \beta_{o}\right)\right|_{0} ^{1} \int \vartheta_{j} \psi_{p}^{\prime \prime} d \theta$

$+\left.\bar{\beta}_{9}\left(\vartheta_{j} \psi_{p}^{\prime}\right)\right|_{0} ^{2 \pi} \int \chi_{i} \beta_{0}^{\prime} d \xi$

$\bar{F}_{u p, b c}=\left.\bar{\beta}_{6}\left(\beta_{r}\right)\right|_{0} ^{1} \int \psi_{s} d \theta$
$(M)_{v H}^{v}=\iint\left(\begin{array}{c}\phi_{k} \phi_{q} \alpha_{l} \alpha_{g}-\mu^{2} \phi_{k} \phi_{\mathrm{q}}^{\prime \prime} \alpha_{l} \alpha_{g} \\ -\mu^{2} m_{0}^{2} \phi_{k} \phi_{q} \alpha_{l} \alpha_{g}^{\prime \prime}\end{array}\right) d \xi d \theta$

$(K)_{v H}^{u}=$

$-\iint\left(\beta_{7} \phi_{k} \chi_{i} \alpha_{l} \vartheta_{j}^{\prime \prime}+\beta_{8} \phi_{k} \chi_{i}^{\prime} \alpha_{l} \vartheta_{j}^{\prime}\right) d \xi d \theta$

$\left(K_{b c}\right)_{v H}^{u}=\left.\bar{\beta}_{10}\left(\phi_{k} \chi_{i}\right)\right|_{0} ^{1} \int \alpha_{l} \vartheta_{j}^{\prime} d \theta$

$+\left.\bar{\beta}_{13}\left(\alpha_{l} \vartheta_{j}\right)\right|_{0} ^{2 \pi} \int \phi_{k} \chi_{\mathrm{i}}^{\prime} d \xi$

$(K)_{v H}^{v}=$

$-\iint\left(\beta_{9} \phi_{k} \phi_{q}^{\prime \prime} \alpha_{l} \alpha_{g}+\beta_{10} \phi_{k} \phi_{q} \alpha_{l} \alpha_{g}^{\prime \prime}\right) d \xi d \theta$

$\left(K_{b c}\right)_{v H}^{v}=\left.\bar{\beta}_{11}\left(\phi_{k} \phi_{q}^{\prime}\right)\right|_{0} ^{l} \int_{0}^{2 \pi} \alpha_{l} \alpha_{g} d \theta$

$+\left.\bar{\beta}_{14}\left(\alpha_{l} \alpha_{g}^{\prime}\right)\right|_{0} ^{2 \pi} \int \phi_{k} \phi_{q} d \xi$

$(K)_{v H}^{w}=$

$-\iint\left(\begin{array}{c}\beta_{11} \phi_{k} \beta_{o} \alpha_{l} \psi_{p}^{\prime}+\beta_{12} \phi_{k} \beta_{0}^{\prime \prime} \alpha_{l} \psi_{p}^{\prime} \\ +\beta_{13} \phi_{k} \beta_{o} \alpha_{l} \psi_{p}^{\prime \prime \prime}\end{array}\right) d \xi d \theta$

$\left(K_{b c}\right)_{v H}^{w}=\left.\bar{\beta}_{12}\left(\phi_{k} \beta_{0}^{\prime}\right)\right|_{0} ^{1} \int_{0}^{2 \pi} \alpha_{l} \psi_{p}^{\prime} d \theta$

$+\bar{\beta}_{15}\left(\left.\alpha_{l} \psi_{p}\right|_{0} ^{2 \pi}\right) \int_{0}^{l} \phi_{k} \beta_{o} d \xi$

$+\bar{\beta}_{16}\left(\left.\alpha_{l} \psi_{p}\right|_{0} ^{2 \pi}\right) \int_{0}^{l} \phi_{k} \beta_{0}^{\prime \prime} d \xi$

$+\bar{\beta}_{17}\left(\left.\alpha_{l} \psi_{p}^{\prime \prime}\right|_{0} ^{2 \pi}\right) \int_{0}^{l} \phi_{k} \beta_{o} d \xi$

$\bar{F}_{v p, b c}=\left.\bar{\beta}_{18}\left(\alpha_{l}\right)\right|_{0} ^{2 \pi} \int \phi_{k} d \xi$

$(M)_{w H}^{w}=\iint\left(\begin{array}{c}\beta_{r} \beta_{o} \psi_{s} \psi_{p}-\mu^{2} \beta_{r} \beta_{o}^{\prime \prime} \psi_{s} \psi_{p} \\ -\mu^{2} m_{0}^{2} \beta_{r} \beta_{o} \psi_{s} \psi_{p}^{\prime \prime}\end{array}\right) d \xi d \theta$

$(C)_{w}^{w}=$

$\iint \bar{C}_{w}\left(\begin{array}{c}\beta_{r} \beta_{o} \psi_{s} \psi_{p}-\mu^{2} \beta_{r} \beta_{o}^{\prime \prime} \psi_{s} \psi_{p} \\ -\mu^{2} m_{0}^{2} \beta_{r} \beta_{o} \psi_{s} \psi_{p}^{\prime \prime}\end{array}\right) d \xi d \theta$

$(K)_{w H}^{u}=$

$-\iint\left(\begin{array}{c}\beta_{14} \beta_{o} \chi_{\mathrm{i}}^{\prime} \psi_{p} \vartheta_{j}+\beta_{15} \beta_{o} \chi_{\mathrm{i}}^{\prime \prime \prime} \psi_{p} \vartheta_{j} \\ +\beta_{16} \beta_{o} \chi_{\mathrm{i}}^{\prime} \psi_{p} \vartheta_{j}^{\prime \prime}\end{array}\right) d \xi d \theta$ 


$$
\begin{aligned}
& \left(K_{b c}\right)_{w H}^{u}=\left.\bar{\beta}_{19}\left(\beta_{o} \chi_{\mathrm{i}}^{\prime \prime}\right)\right|_{0} ^{1} \int_{0}^{2 \pi} \psi_{p} \vartheta_{j} d \theta \\
& +\left.\bar{\beta}_{20}\left(\beta_{o} \chi_{i}\right)\right|_{0} ^{1} \int_{0}^{2 \pi} \psi_{p} \vartheta_{j}^{\prime \prime} d \theta \\
& +\left.\bar{\beta}_{31}\left(\beta_{o} \chi_{\mathrm{i}}^{\prime}\right)\right|_{0} ^{1} \int_{0}^{2 \pi} \psi_{p} \vartheta_{j} d \theta \\
& +\left.\bar{\beta}_{40}\left(\beta_{o} \chi_{i}\right)\right|_{0} ^{1} \int_{0}^{2 \pi} \psi_{p} \vartheta_{j}^{\prime} d \theta \\
& +\left.\bar{\beta}_{25}\left(\psi_{p} \vartheta_{j}^{\prime}\right)\right|_{0} ^{2 \pi} \int \beta_{o} \chi_{\mathrm{i}}^{\prime} d \xi \\
& +\left.\bar{\beta}_{37}\left(\psi_{p} \vartheta_{j}^{\prime}\right)\right|_{0} ^{2 \pi} \int \beta_{o} \chi_{i} d \xi \\
& +\left.\bar{\beta}_{43}\left(\psi_{p} \vartheta_{j}\right)\right|_{0} ^{2 \pi} \int \beta_{o} \chi_{\mathrm{i}}^{\prime} d \xi \\
& (K)_{w H}^{v}= \\
& -\iint\left(\begin{array}{c}
\beta_{17} \beta_{o} \phi_{k} \psi_{p} \alpha_{l}^{\prime}+\beta_{18} \beta_{o} \phi_{k}^{\prime \prime} \psi_{p} \alpha_{l}^{\prime} \\
+\beta_{19} \beta_{o} \phi_{k} \psi_{p} \alpha_{l}^{\prime \prime \prime}
\end{array}\right) d \xi d \theta \\
& \left(K_{b c}\right)_{w H}^{v}=\left.\bar{\beta}_{21}\left(\beta_{o} \phi_{k}^{\prime}\right)\right|_{0} ^{1} \int_{0}^{2 \pi} \psi_{p} \alpha_{l}^{\prime} d \theta \\
& +\left.\bar{\beta}_{32}\left(\beta_{o} \phi_{k}\right)\right|_{0} ^{1} \int_{0}^{2 \pi} \psi_{p} \alpha_{l}^{\prime} d \theta \\
& +\left.\bar{\beta}_{41}\left(\beta_{o} \phi_{k}^{\prime}\right)\right|_{0} ^{1} \int_{0}^{2 \pi} \psi_{p} \alpha_{l} d \theta \\
& +\left.\bar{\beta}_{26}\left(\psi_{p} \alpha_{l}\right)\right|_{0} ^{2 \pi} \int \beta_{o} \phi_{k}^{\prime \prime} d \xi \\
& +\left.\bar{\beta}_{27}\left(\psi_{p} \alpha_{l}^{\prime \prime}\right)\right|_{0} ^{2 \pi} \int \beta_{o} \phi_{k} d \xi \\
& +\left.\bar{\beta}_{38}\left(\psi_{p} \alpha_{l}\right)\right|_{0} ^{2 \pi} \int \beta_{o} \phi_{k}^{\prime} d \xi \\
& +\left.\bar{\beta}_{44}\left(\psi_{p} \alpha_{l}^{\prime}\right)\right|_{0} ^{2 \pi} \int \beta_{o} \phi_{k} d \xi \\
& (K)_{w H}^{w}= \\
& -\iint\left(\begin{array}{c}
\beta_{20} \beta_{o} \beta_{r} \psi_{s} \psi_{p}+\beta_{21} \beta_{o} \beta_{r}^{\prime \prime} \psi_{s} \psi_{p} \\
+\beta_{22} \beta_{r} \beta_{o} \psi_{s} \psi_{p}^{\prime \prime}+\beta_{23} \beta_{o} \beta_{r}^{\prime \prime \prime} \psi_{s} \psi_{p} \\
+\beta_{24} \beta_{o} \beta_{r}^{\prime \prime} \psi_{s} \psi_{p}^{\prime \prime}+\beta_{25} \beta_{o} \beta_{r} \psi_{s} \psi_{p}^{\prime \prime \prime \prime}
\end{array}\right) d \xi d \theta \\
& (\bar{K})_{w H}= \\
& \bar{k}_{w} \iint\left(\begin{array}{c}
\beta_{r} \beta_{o} \psi_{s} \psi_{p}-\mu^{2} \beta_{r} \beta_{o}^{\prime \prime} \psi_{s} \psi_{p} \\
-\mu^{2} m_{0}^{2} \beta_{r} \beta_{o} \psi_{s} \psi_{p}^{\prime \prime}
\end{array}\right) d \xi d \theta \\
& (\bar{K})_{p H}= \\
& -\bar{k}_{p} \iint\left(\begin{array}{c}
\beta_{o} \beta_{r}^{\prime \prime} \psi_{s} \psi_{p}+m_{0}^{2} \beta_{o} \beta_{r} \psi_{s} \psi_{p}^{\prime \prime} \\
-\mu^{2} \beta_{r} \beta_{o}^{\prime \prime \prime \prime} \psi_{s} \psi_{p}-\mu^{2} m_{0}^{2} \beta_{o} \beta_{r}^{\prime \prime} \psi_{s} \psi_{p}^{\prime \prime} \\
-\mu^{2} m_{0}^{2} \beta_{r} \beta_{o}^{\prime \prime} \psi_{s} \psi_{p}^{\prime \prime}-\mu^{2} m_{0}^{4} \beta_{o} \beta_{r} \psi_{s} \psi_{p}^{\prime \prime \prime \prime}
\end{array}\right) d \xi d \theta \\
& \bar{F}_{w p H}=\beta_{26} \iint\left(\beta_{r} \psi_{s}\right) d \xi d \theta \\
& \left(K_{b c}\right)_{w H}^{w}=\left.\bar{\beta}_{22}\left(\beta_{o} \beta_{r}^{\prime}\right)\right|_{0} ^{1} \int_{0}^{2 \pi} \psi_{s} \psi_{p} d \theta \\
& +\left.\bar{\beta}_{23}\left(\beta_{o} \beta_{r}^{\prime \prime \prime}\right)\right|_{0} ^{1} \int_{0}^{2 \pi} \psi_{s} \psi_{p} d \theta \\
& +\left.\bar{\beta}_{24}\left(\beta_{o} \beta_{r}^{\prime}\right)\right|_{0} ^{1} \int_{0}^{2 \pi} \psi_{s} \psi_{p}^{\prime \prime} d \theta \\
& +\left.\bar{\beta}_{33}\left(\beta_{o} \beta_{r}\right)\right|_{0} ^{1} \int_{0}^{2 \pi} \psi_{s} \psi_{p} d \theta \\
& +\left.\bar{\beta}_{34}\left(\beta_{o} \beta_{r}^{\prime \prime}\right)\right|_{0} ^{1} \int_{0}^{2 \pi} \psi_{s} \psi_{p} d \theta \\
& +\left.\bar{\beta}_{35}\left(\beta_{o} \beta_{r}\right)\right|_{0} ^{1} \int_{0}^{2 \pi} \psi_{s} \psi_{p}^{\prime \prime} d \theta \\
& +\left.\bar{\beta}_{42}\left(\beta_{o} \beta_{r}^{\prime}\right)\right|_{0} ^{1} \int_{0}^{2 \pi} \psi_{s} \psi_{p}^{\prime} d \theta \\
& +\left.\bar{\beta}_{28}\left(\psi_{s} \psi_{p}^{\prime}\right)\right|_{0} ^{2 \pi} \int \beta_{o} \beta_{r} d \xi \\
& +\left.\bar{\beta}_{29}\left(\psi_{s} \psi_{p}^{\prime \prime \prime}\right)\right|_{0} ^{2 \pi} \int \beta_{o} \beta_{r} d \xi \\
& +\left.\bar{\beta}_{30}\left(\psi_{s} \psi_{p}^{\prime}\right)\right|_{0} ^{2 \pi} \int \beta_{o} \beta_{r}^{\prime \prime} d \xi \\
& +\left.\bar{\beta}_{39}\left(\psi_{s} \psi_{p}^{\prime}\right)\right|_{0} ^{2 \pi} \int \beta_{o} \beta_{r}^{\prime} d \xi
\end{aligned}
$$




$$
\begin{aligned}
& +\left.\bar{\beta}_{45}\left(\psi_{s} \psi_{p}\right)\right|_{0} ^{2 \pi} \int \beta_{o} \beta_{r} d \xi \\
& +\left.\bar{\beta}_{46}\left(\psi_{s} \psi_{p}\right)\right|_{0} ^{2 \pi} \int \beta_{o} \beta_{r}^{\prime \prime} d \xi \\
& +\left.\bar{\beta}_{47}\left(\psi_{s} \psi_{p}^{\prime \prime}\right)\right|_{0} ^{2 \pi} \int \beta_{o} \beta_{r} d \xi \\
& \bar{F}_{w p, b c}= \\
& \left.\bar{\beta}_{36}\left(\beta_{o}\right)\right|_{0} ^{1} \int_{0}^{2 \pi} \psi_{s} d \theta+\left.\bar{\beta}_{48}\left(\psi_{s}\right)\right|_{0} ^{2 \pi} \int \beta_{o} d \xi
\end{aligned}
$$

\section{References}

[1] Zhang SJ, Xia R, Lebrun L, Anderson D, Shrout TR. Piezoelectric materials for high power, high temperature applications. Materials Letters 2005; 59(27): 3471-5.

[2] Manbachi A, Cobbold RSC. Development and application of piezoelectric materials for ultrasound generation and detection. Ultrasound 2011; 11(4): 187-96.

[3] Jalili N. Piezoelectric-Based Vibration Control: From Macro to Micro/Nano Scale Systems. New York: Springer; 2010.

[4] Mindlin RD, Tiersten HF. Effects of couplestresses in linear elasticity. Archive for Rational Mechanics and Analysis 1962; 11(1): 415-48.

[5] Eringen AC. Nonlocal polar elastic continua. International Journal of Engineering Science 1972; 10(1): 1-16.

[6] Eringen AC. Nonlocal Continuum Field Theories. New York: Springer-Verlag; 2002.

[7] Mindlin RD, Eshel NN. on first strain-gradient theories in linear elasticity. International Journal of Solids and Structures 1968; 4(1): 109-24.

[8] Gurtin ME, Murdoch AI. A continuum theory of elastic material surface. Archive for Rational Mechanics and Analysis 1975; 57(4): 291-323.

[9] Gurtin ME, Murdoch AI. Surface stress in solids. International Journal of Solids and Structures 1978; 14(6): 431-40.

[10] Fang XQ, Liu JX, Gupta V. Fundamental formulations and recent achievements in piezoelectric nano-structures: a review. Nanoscale 2017; 5(5): 1716-26.

[11] Zhu CS, Fang XQ, Liu JX. Surface energy effect on buckling behavior of the functionally graded nano-shell covered with piezoelectric nano-layers under torque. International Journal of Mechanical Sciences 2017; 133: 662-673.

[12] Fang XQ, Zhu CS, Liu JX, Zhao J. Surface energy effect on nonlinear buckling and postbuckling behavior of functionally graded piezoelectric cylindrical nanoshells under lateral pressure. Materials Research Express 2018; 5(4): 045017.

[13] Fang XQ, Zhu CS, Liu JX. Surface energy effect on free vibration of nano-sized piezoelectric double-shell structures. Physica B: Condensed Matter 2018; 529(15): 41-56.

[14] Fang XQ, Zhu CS, Liu JX. Surface energy effect on nonlinear free vibration behavior of orthotropic piezoelectric cylindrical nanoshells. European Journal of Mechanics A/Solids 2017; 66: 423-432.

[15] Gholami R, Darvizeh A, Ansari R, Pourashraf T. Analytical Treatment of the Size-Dependent Nonlinear Postbuckling of Functionally Graded Circular Cylindrical Micro-/Nano-Shells. Iranian Journal of Science and Technology, Transactions of Mechanical Engineering. 2017; DOI 10.1007/s40997-017-0080-6.

[16] Sahmani S, Aghdam MM. Boundary Layer Modeling of Nonlinear Axial Buckling Behavior of Functionally Graded Cylindrical Nanoshells Based on the Surface Elasticity Theory. Iranian Journal of Science and Technology, Transactions of Mechanical Engineering. 2017; DOI 10.1007/s40997-0170092-2.

[17] Gheshlaghi B, Hasheminejad SM. Surface effects on nonlinear free vibration of nanobeams. Composites Part B: Engineering 2011; 42(4): 934-7.

[18] Wang L. Vibration Analysis of FluidConveying Nanotubes with Consideration of Surface Effects. Physica E: Low-Dimensional Systems and Nanostructures 2010; 43: 437439.

[19] Zhang LL, Liu JX, Fang XQ. Size-dependent dispersion characteristics in piezoelectric 
nanoplates with surface effects. Physica E: Low-Dimensional Systems and Nanostructures 2014; 57: 169-174.

[20] Wang KF, Wang BL. Effects of residual surface stress and surface elasticity on the nonlinear free vibration of nanoscale plates. Journal of Applied Physics 2012; 112(1): 013520-5.

[21] Rahimi GH, Ansari R, emmatnezhad M. Vibration of functionally graded cylindrical shells with ring support. Scientia Iranica $B$ 2011; 18 (6): 1313-1320.

[22] Avramov KV. Nonlinear vibrations characteristics of single-walled carbon nanotubes by nonlocal elastic shell model. International Journal of Non-Linear Mechanics 2018; 43: 109-117.

[23] Saadatnia Z, Esmailzadeh E. Nonlinear harmonic vibration analysis of fluidconveying piezoelectric-layered nanotubes. Composites Part B: Engineering 2017; 123(15): 193-209.

[24] Leissa AW. Vibration of Shells. NASA SP 288, US Government Printing Office, 1973, Reprinted by the Acoustical Society of America; 1993.

[25] Liew KM, Lim CW, Kitipornchai S. Vibration of shallow shells: a review with bibliography. Applied Mechanics Reviews 1997; 50(8):431444.

[26] Loy CT, Lam KY, Shu C. Analysis of cylindrical shells using generalized differential quadrature. Shock and Vibration 1997; 4(3):193-198.

[27] Hashemi Kachapi Sayyid H., Dardel M., Mohamadi daniali H., Fathi A., Pull-in instability and nonlinear vibration analysis of electrostatically piezoelectric nanoresonator with surface/interface effects, Thin-Walled Structures, 143, 106210, 2019.

[28] Hashemi Kachapi Sayyid H., Dardel M., Mohamadi daniali H., Fathi A., Nonlinear dynamics and stability analysis of piezo-visco medium nanoshell resonator with electrostatic and harmonic actuation, Applied Mathematical Modelling, DOI:10.1016/j.apm.2019.05.035, 2019.
[29] Hashemi Kachapi Sayyid H., Dardel M., Mohamadi daniali H., Fathi A., Nonlinear vibration and stability analysis of doublewalled piezoelectric nanoresonator with nonlinear van der Waals and electrostatic excitation. Journal of Vibration and Control, DOI: 10.1177/1077546319889858, 2019.

[30] Hashemi Kachapi Sayyid H., Dardel M., Mohamadi daniali H., Fathi A., Effects of surface energy on vibration characteristics of double-walled piezo-viscoelastic cylindrical nanoshell, Proceedings of the Institution of Mechanical Engineers, Part C: Journal of Mechanical Engineering Science, DOI: 10.1177/0954406219845019, 2019.

[31] Hashemi Kachapi Sayyid H., Mohamadi daniali H., Dardel M., Fathi A., The effects of nonlocal and surface/interface parameters on nonlinear vibrations of piezoelectric nanoresonator, Journal of Intelligent Material Systems and Structures, DOI: 10.1177/1045389X19898756, 2020.

[32] Hashemi Kachapi Sayyid H., Nonlinear vibration and stability analysis of piezo-harmoelectrostatic nanoresonator based on surface/interface and nonlocal strain gradient effects, Journal of the Brazilian Society of Mechanical Sciences and Engineering, DOI: 10.1007/s40430-020-2173-1, 42(107) 2020.

[33] Hashemi Kachapi Sayyid H., Surface/interface approach in pull-in instability and nonlinear vibration analysis of fluid-conveying piezoelectric nanosensor, Mechanics Based Design of Structures and Machines, DOI: 10.1080/15397734.2020.1725566, 2020.

[34] Hashemi Kachapi Sayyid H., Nonclassical vibration analysis of piezoelectric nanosensor conveying viscous fluid, WSEAS Transactions on Applied and Theoretical Mechanics, 14, 2019.

[35] Hashemi Kachapi Sayyid H., Dardel M., Mohamadi daniali H., Fathi A., Non-linear Frequency Response and Stability Analysis of Piezoelectric Nanoresonator Subjected to Electrostatic Excitation, International Journal of Nonlinear Sciences and Numerical Simulation, DOI: 10.1515/ijnsns-2018-0269, 2019. 
[36] Hashemi Kachapi Sayyid H., Dardel M., Mohamadi daniali H., Fathi A., Nonlinear Dynamic Response and Frequency Analysis of the FG-PCNS With Surface Energy Effects, Romanian Journal of Acoustics and Vibration, 16(1) 15-31, 2019.

[37] Donnell LH. Beam, Plates and Shells. New York: McGraw-Hill; 1976.

[38] Amabili M. Nonlinear Vibrations and Stability of Shells and Plates. New York: Cambridge University Press; 2008.

[39] Torabi J, Kiani Y, Eslami MR. Linear thermal buckling analysis of truncated hybrid FGM conical shells. Composites Part B 2013; 50: 265-72.

[40] Lu P, He LH, Lee HP, Lu C. Thin plate theory including surface effects. International Journal of Solids and Structure 2006; 43(16): 4631-47.

[41] Mosallaie Barzoki AA, Ghorbanpour Arani A, Kolahchi R, Mozdianfard MR, Loghman A. Nonlinear buckling response of embedded piezoelectric cylindrical shell reinforced with BNNT under electro-thermo-mechanical loadings using HDQM. Composites: Part B 2013; 44: 722-727.

[42] Jafari AA, Khalili SMR, Tavakolian M. Nonlinear vibration of functionally graded cylindrical shells embedded with a piezoelectric layer. Thin- Walled Structures 2014; 79: 8-15.

[43] Sudip Basack, Ghritartha Goswami, Prandeep Deka, Partha Pratim Borah, Nikos Mastorakis, Analysis and Control of Flow Parameters through Sluice Gate in Dam, International Journal of Mechanics, NAUN, pp. 22-27, Volume 14, 2020

[44] Ouadie Koubaiti, Ahmed Elkhalfi, Jaouad ElMekkaoui, Nikos Mastorakis, Solving the Problem of Constraints Due to Dirichlet Boundary Conditions in the Context of the Mini Element Method, International Journal of Mechanics, NAUN, pp. 12-21 Volume 14, 2020

[45] Rao SS. Vibration of Continuous Systems. New Jersey: John Wiley \& Sons; 2007.

[46] Ke LL, Wang YS, Reddy JN. Thermo-electromechanical vibration of size-dependent piezoelectric cylindrical nanoshells under various boundary conditions. Composite Structures 2014; 116(1): 626-636. 